\title{
Modeling the spectral energy distribution of ULIRGs
}

\section{The radio spectra ${ }^{\star}$}

\author{
M. S. Clemens ${ }^{1}$, O. Vega ${ }^{1,2}$, A. Bressan ${ }^{1,2,3}$, G. L. Granato ${ }^{1,3}$, L. Silva ${ }^{4}$, and P. Panuzzo ${ }^{1,5}$ \\ 1 INAF - Osservatorio Astronomico di Padova, Vicolo dell'Osservatorio 5, 35122 Padova, Italy \\ e-mail: marcel.clemens@oapd.inaf.it \\ 2 INAOE, Luis Enrique Erro 1, 72840 Tonantzintla, Puebla, Mexico \\ 3 SISSA, Strada Costiera, 34131 Trieste, Italy \\ 4 INAF - Osservatorio Astronomico di Trieste, via Tiepolo 11, 34131 Trieste, Italy \\ 5 Laboratoire AIM, CEA/DSM - CNRS - Université Paris Diderot, DAPNIA/Service d'Astrophysique, Bât. 709, CEA-Saclay, \\ 91191 Gif-sur-Yvette Cedex, France
}

Received 2 February 2007 / Accepted 8 October 2007

\begin{abstract}
Aims. We aim to constrain new starburst/AGN models of IRAS bright galaxies via their spectral energy distribution from the nearinfrared to the radio. To this end, we determine the radio spectra for a sample of 31 luminous and ultraluminous IRAS galaxies (LIRGs/ULIRGs).

Methods. We present here new high frequency VLA observations at $22.5 \mathrm{GHz}$ and $8.4 \mathrm{GHz}$ and also derive fluxes at other radio frequencies from archival data. Together with radio data from the literature, we construct the radio spectrum for each source. In the selection of data we have made every effort to ensure that these fluxes neither include contributions from nearby objects, nor underestimate the flux due to high interferometer resolution.

Results. From our sample of well-determined radio spectra we find that very few have a straight power-law slope. Although some sources show a flattening of the radio spectral slope at high frequencies, the average spectrum shows a steepening of the radio spectrum from 1.4 to $22.5 \mathrm{GHz}$. This is unexpected, because in sources with high rates of star formation, we expect that flat spectrum, free-free emission will make a significant contribution to the radio flux at higher radio frequencies. Despite this trend, the radio spectral indices between 8.4 and $22.5 \mathrm{GHz}$ are flatter for sources with higher values of the far-infrared (FIR)-radio flux density ratio, $q$, when this is calculated at $8.4 \mathrm{GHz}$. Therefore, sources that are deficient in radio emission relative to FIR emission (presumably younger sources) have a larger thermal component to their radio emission. However, we find no correlation between the radio spectral index between 1.4 and $4.8 \mathrm{GHz}$ and $q$ at $8.4 \mathrm{GHz}$. Because the low frequency spectral index is affected by free-free absorption, and this is a function of source size for a given mass of ionised gas, this is evidence that the ionised gas in ULIRGs shows a range of densities.

Conclusions. The youngest LIRGs and ULIRGs are characterised by flatter average radio spectral indices from 1.4 to $22.5 \mathrm{GHz}$, and by a larger contribution to their high frequency, radio spectra from free-free emission. However, the youngest sources are not those that have the greatest free-free absorption at low radio frequencies. The sources in which the effects of free-free absorption are strongest are instead the most compact sources. Although these have the warmest FIR colours, they are not necessarily the youngest sources.
\end{abstract}

Key words. dust, extinction - galaxies: active - infrared: galaxies - radio continuum: galaxies

\section{Introduction}

The nature of the power source for ULIRGs remains a much debated issue. The presence of either an AGN or a starburst in a given source is not evidence that one or the other is the principal power source for the infrared luminosity. The observation that ULIRGs fall on the same FIR-radio correlation as star-forming galaxies (e.g. Sopp et al. 1990) was taken as evidence that these sources are powered predominantly by star formation. Analogous correlations exist between the Br $\gamma$ luminosity and far-infrared (FIR) luminosity (Goldader et al. 1997) and dense molecular gas mass and FIR luminosity (Gao \& Solomon 2004). However, evidence that AGN activity is not independent of starburst activity has made such conclusions less secure.

^ Appendix A, Figs. 3-5 are only available in electronic form at http://www . aanda.org
Farrah et al. (2003) show, in fact, that AGN and starburst luminosities are correlated over a wide range of IR luminosities. As ULIRGs may commonly host both an AGN and a starburst, their relative contributions need to be quantified.

Apart from the rarity of ULIRGs (there is no example closer than Arp 220 at $72 \mathrm{Mpc}$ ), their study is made difficult by the large and uncertain extinctions toward their centres. In many cases the extinction is not even negligible in the mid-infrared; it is likely that $\mathrm{H} \alpha$ emission detected from a ULIRG does not originate from the deeply embedded regions where the FIR luminosity is generated. The less extinguished, outer regions of a ULIRG may host a certain rate of star formation that causes a certain amount of $\mathrm{H} \alpha$ emission but this could be quite independent of whether it hosts a central starburst or an AGN. Murphy et al. (2001) find very few broad near-infrared lines in ULIRGs which would be the signature of an AGN, but note that very high extinctions could exclude their detection even at $2 \mu \mathrm{m}$. 
Flores et al. (2004) recently showed that even when corrected by $\mathrm{H} \gamma$ data, the $\mathrm{H} \alpha$ luminosity can underestimate the star formation rate by a factor of 2 for ULIRGs. Both Goldader et al. (1995) and Valdés et al. (2005) find that near-infrared recombination lines $(\mathrm{Pa} \alpha$ and $\mathrm{Br} \gamma)$ are under-luminous in ULIRGs compared to what would be expected from a starburst of similar bolometric luminosity. Their results indicate that even near-infrared observations may not penetrate the most obscured regions in these sources. This picture is consistent with the conclusions of Poggianti et al. (2001) that the extinction in the centre of ULIRGs is a function of the age of the star formation episode, with younger stars being more heavily extinguished, as introduced by Silva et al. (1998) to describe star-forming regions. The lack of correlation between super star clusters and HII regions found in luminous infrared galaxies by Alonso-Herrero et al. (2002) also supports a scenario of age-dependent extinction. In order to estimate the star formation rate in these sources, tracers that do not suffer extinction are preferred.

Perhaps the most direct way to determine the power source in ULIRGs is via radio recombination lines which trace the ionised gas without suffering extinction. Observations of several lines between 1.4 and $207 \mathrm{GHz}$ of Arp 220 have been used by Anantharamaiah et al. (2000) to show that this source is powered by star formation. Unfortunately, very few ULIRGs are accessible observationally to such observations with existing instruments.

One approach which has been used to distinguish between AGN and starburst power sources in ULIRGs has been to search for very compact radio continuum emission towards the nucleus or nuclei. Interestingly, radio sources of similar physical sizes have been identified as both AGN and compact starbursts, depending on the resolution of the observations. Nagar et al. (2003) use $15 \mathrm{GHz}$ radio continuum data with a resolution of 150 mas to investigate the nature of 83 ULIRGs. One argument used by these authors to conclude that most ULIRGs are AGN powered is the compactness of the radio sources detected. The resolution of their data corresponds to $420 \mathrm{pc}$ at the median redshift of their sample. However, the supernovae ( $\mathrm{SNe}$ ) detected in the NW nucleus of Arp 220 (Smith et al. 1998; Lonsdale et al. 2006) are within a region $0.2 \times 0 . \prime 4(75 \times 150 \mathrm{pc})$. Both of these studies found that no AGN is necessary to explain the IR luminosity of Arp 220. As long as the brightness temperature does not exceed $10^{6} \mathrm{~K}$ the compactness of radio nuclei alone does not support an AGN hypothesis.

Rather than look at the radio morphology, we examine the spectrum of the radio emission. In star-forming galaxies the radio spectrum is made up of two components: a non-thermal, synchrotron component and a thermal, "Bremsstrahlung" component, often referred to as "free-free" emission. At $\mathrm{GHz}$ frequencies, the synchrotron spectrum, $S_{v} \propto$ $v^{\alpha}$, has a typical power-law slope, $\alpha \sim-0.8$, while the free-free emission has a much flatter slope, $\alpha=-0.1$. Free-free emission thus makes a larger contribution at higher radio frequencies, so that the radio spectrum flattens at high frequencies. Half of the radio flux is expected to be of thermal origin at $\sim 20 \mathrm{GHz}$ (Condon 1992). Towards lower radio frequencies (below $1 \mathrm{GHz}$ in non-starburst galaxies) the free-free optical depth becomes large and the (synchrotron-dominated) radio spectrum shows a sharp decline due to free-free absorption (see Fig. 4 of Condon 1992). In sources with very intense star formation, free-free absorption may be expected to occur at higher frequencies than in quiescent objects; the $1.4 \mathrm{GHz}$ fluxes from such objects are probably affected.
In principle, a measure of the thermal radio flux from a ULIRG would be an excellent measure of the star formation rate, because, being emitted by the same gas from which recombination lines originate, it traces gas ionised by young stars. Absorption of ionising photons by dust may complicate this picture slightly (Valdés et al. 2005) but the main difficulty is in estimating the thermal fraction. Although the fractional contribution from thermal emission to the total radio flux increases with frequency, the radio emission from ULIRGs is dominated by synchrotron radiation even at frequencies above $15 \mathrm{GHz}$. As we will see later, the radio spectral indices around $15 \mathrm{GHz}$ are normally much steeper than the -0.1 expected from a purely thermal spectrum. The motivation for the $22 \mathrm{GHz}$ observations described here was to better constrain the thermal radio flux.

Because the thermal radio flux provides an estimate of the present star-formation rate, it is important in fitting starburst/AGN models to the spectral energy distribution (SED) from the near-infrared to the radio. Bressan et al. (2002) showed that deviations from the FIR/radio correlation could be used to derive the evolutionary status of a compact starburst. Such deviations are expected in bursts of short duration because at early times (a few $\times 10^{6} \mathrm{yr}$ ) even the most massive stars formed in the burst will not have ended their lives as SNe and no excess synchrotron emission should result from the starburst activity. Such young sources then, should have a FIR/radio ratio above the mean FIR-radio correlation and should have flatter radio slopes due to the greater ratio of thermal/synchrotron emission. In Prouton et al. (2004) we illustrated the power of this technique with special emphasis on high frequency radio data. A second paper, Vega et al. (2007), hereafter Paper II, will be concerned with the model fits to the SEDs from the near-infrared to the radio.

Here we present new radio data at 22.5 and $8.4 \mathrm{GHz}$, as well as archival radio data which have been re-reduced, and radio fluxes from the literature. The result is a set of 31, well-sampled radio spectra for infrared-luminous galaxies. In Sect. 2 we describe the sample selection and in Sect. 3 the data acquisition and reduction. In Sect. 4 we describe the radio spectra in detail, and in Sect. 5, the implications of these spectra when compared to FIR fluxes and the FIR-radio flux ratio, $q$. Conclusions are reached in Sect. 6. In an appendix we comment on individual objects and present the radio spectra in tabular form.

\section{Sample selection}

We have based our sample on that of Condon et al. (1991a) who made $8.4 \mathrm{GHz}$ observations of the 40 ULIRGs brighter than $5.25 \mathrm{Jy}$ at $60 \mu \mathrm{m}$ in the IRAS Bright Galaxy Sample. In Prouton et al. (2004) we observed 7 of the ULIRGs observed by Condon et al. but restricted our attention to objects with a ratio of FIR to $1.4 \mathrm{GHz}$ luminosity $q \geq 2.5$, in an attempt to preferentially select young starbursts. Here we have relaxed this restriction on $q$ so as to maximise the sample size and include more diverse objects, and have observed a further 18 sources at $22.5 \mathrm{GHz}$. The remaining sources were either too weak for observation at $22 \mathrm{GHz}$ or were too confused with nearby sources to construct a reliable SED. We also re-observed 7 sources at $8.4 \mathrm{GHz}$ for which the high resolution observations of Condon et al. (1991a) could not provide reliable integrated fluxes. Our final sample is therefore not a FIR flux limited sample like that of Condon et al., but simply contains all those brighter than $5.25 \mathrm{Jy}$ at $60 \mu \mathrm{m}$ for which an integrated $22.5 \mathrm{GHz}$ flux could be reliably obtained.

In addition to the new high frequency observations, we have obtained, either from the literature or from reduction of archival 


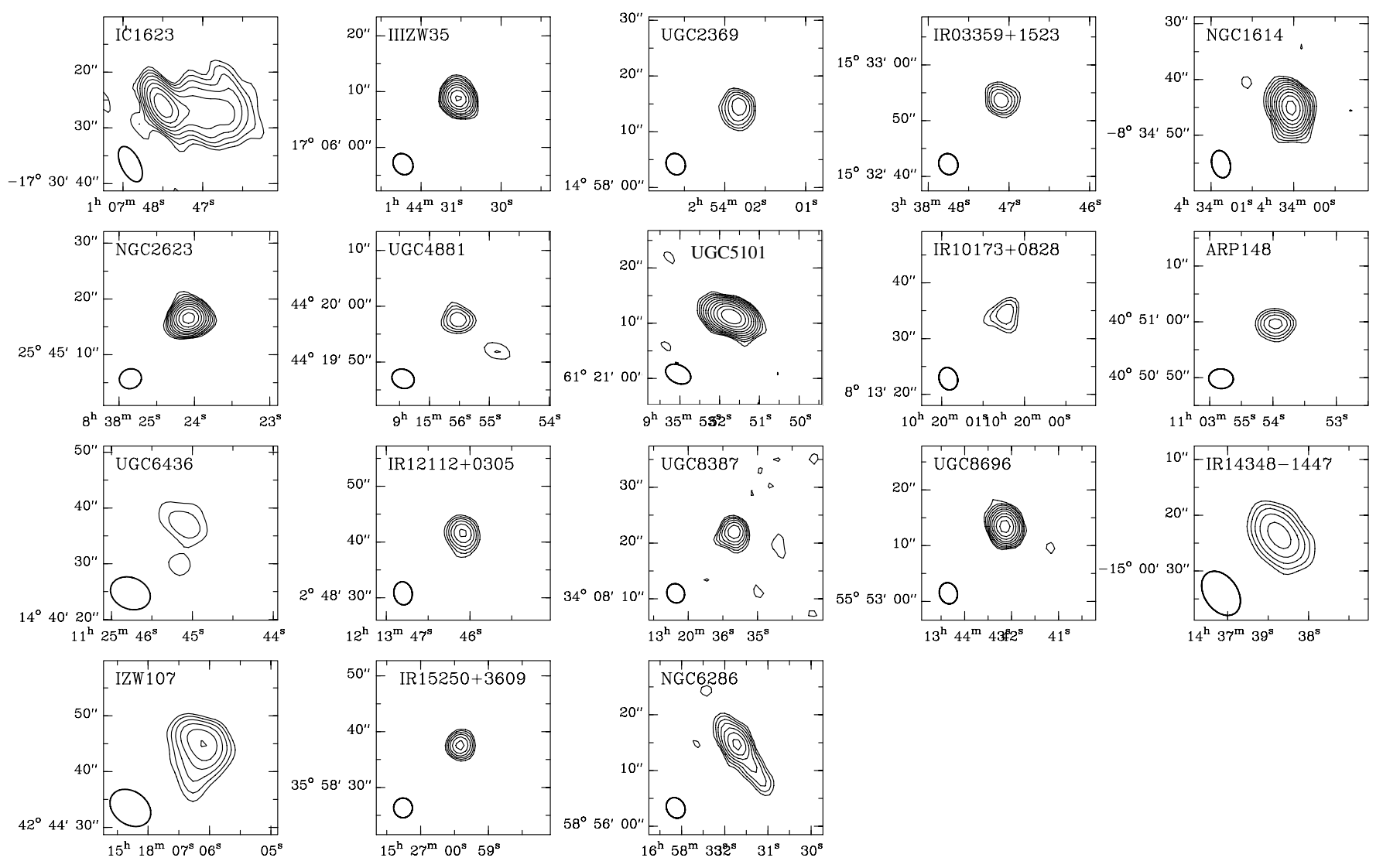

Fig. 1. $22.5 \mathrm{GHz}$ images of the newly observed ULIRGs in our sample. Contour levels begin at $\pm 0.5 \mathrm{mJy}$ beam $^{-1}$ and are spaced by a factor of $2^{1 / 2}$. The synthesised beam is shown in the bottom left of each panel.

VLA data, radio fluxes at as many frequencies as possible. In total, of the 40 ULIRGs of the sample of Condon et al., 31 have radio data at 3 or more frequencies and 29 of these have fluxes at $22.5 \mathrm{GHz}$.

All our sample galaxies are at a redshift $z<0.1$.

\section{Observations and data reduction}

\subsection{New 22.5 and $8.4 \mathrm{GHz}$ data}

The 22.5 GHz observations were carried out on 2004, August 13 and 14 with the VLA in D-configuration. Total integration times for the sources were between 16 and 24 min, with scans onsource being interleaved between scans of phase calibrators every $8 \mathrm{~min}$. Before each new source was observed, a pointing scan was carried out on the phase calibrator at $8.4 \mathrm{GHz}$ to determine a pointing correction. 3C 286 was observed as an absolute flux calibrator on 13 August and 3C 48 on 15 August.

The weather conditions on August 13 were not ideal for high frequency radio observations, data were taken in the presence of thunder storms. As water vapour affects the measured flux for a given source at $22 \mathrm{GHz}$, and the flux calibrator may have been observed through a different cloud density than some of the phase calibrators, the bootstrapping of the phase calibrator fluxes to those of the flux calibrators is not guaranteed to work. After flux calibration based on the CLEAN component models for 3C 286 and 3C 48 provided by NRAO the phase calibrator fluxes were within $20 \%$ of those given by the VLA calibrator database. Only in the case of $1310+323$ (the phase calibrator for UGC 8387) was there a larger discrepancy (a factor of 2 less than that given in the database).
Maps were made using "natural" weighting in the uv-plane and in the case of UGC 6436, IZW 107 and IRAS 14348-144 a uv-taper of $30 \mathrm{k} \lambda$ was applied to avoid the possibility of missing flux due to extended but weak structure. The final maps, shown in Fig. 1, had a typical resolution of $4^{\prime \prime}\left(6^{\prime \prime}\right.$ where tapered) and after cleaning had a typical rms noise level of $0.12 \mathrm{mJy} /$ beam. Fluxes were measured by direct integration on the maps. These fluxes (reported in Table 1) have errors which include the rms noise and the flux calibration errors described above.

The $8.44 \mathrm{GHz}$ observations were made on 2005, July 12, 20 and 27 with the C-configuration of the VLA. Integration times of 11 to $14 \mathrm{~min}$ were used and interleaved with observations of nearby phase calibrators. 3C 286 and 3C 48 were used as absolute flux calibrators and, as for the $22 \mathrm{GHz}$ data, calibration made use of CLEAN component models for these sources. Derived fluxes for all phase calibrators agreed with those in the VLA calibrator database to better than $5 \%$. All sources were also self-calibrated to correct for phase only. Maps were made by natural weighting the uv-data and cleaned in the standard way. Resolution and rms in the resulting maps were typically $3^{\prime \prime}$ and $0.03 \mathrm{mJy}$ beam $^{-1}$. The radio maps for these sources are presented in Fig. 2 and the corresponding integrated fluxes in Table 2.

\subsection{Archival VLA data}

In addition to the above data and fluxes taken from the literature, the VLA archive was searched for data at additional frequencies so as to maximise the coverage of the radio spectrum. Data 
Table 1. 22.5 GHz fluxes derived from the new VLA observations.

\begin{tabular}{cccccc}
\hline \hline Source & $\begin{array}{c}\text { RA } \\
(\mathrm{J} 2000)\end{array}$ & $\begin{array}{c}\text { Dec } \\
(\mathrm{J} 2000)\end{array}$ & $\begin{array}{c}\text { Source flux } \\
(\mathrm{mJy})\end{array}$ & $\begin{array}{c}\text { Phase cal } \\
(\mathrm{J} 2000)\end{array}$ & $\begin{array}{c}\text { Phase cal flux } \\
(\mathrm{Jy})\end{array}$ \\
\hline NGC 34* & 001106.550 & -120626.33 & $7.41 \pm 0.14$ & $2358-103$ & $0.5719 \pm 0.005$ \\
IC 1623 & 010747.49 & -173026.3 & $21.7 \pm 2.0$ & $0050-094$ & $0.805 \pm 0.007$ \\
CGCG436-30* & 012002.722 & +142142.94 & $8.73 \pm 0.31$ & $0121+118$ & $1.177 \pm 0.007$ \\
IRAS 01364-1042* & 013852.870 & -102711.70 & $3.97 \pm 0.19$ & $0141-094$ & $0.7395 \pm 0.003$ \\
IIIZw 35 & 014430.53 & +170608.5 & $9.7 \pm 0.3$ & $0121+118$ & $1.449 \pm 0.011$ \\
UGC 2369 & 025401.81 & +145814.5 & $5.3 \pm 0.3$ & $0238+166$ & $2.041 \pm 0.015$ \\
IRAS 03359+1523 & 033847.12 & +153253.5 & $4.5 \pm 0.3$ & $0325+226$ & $0.768 \pm 0.006$ \\
NGC 1614 & 043400.02 & -083444.98 & $21.0 \pm 1$ & $0423-013$ & $7.480 \pm 0.058$ \\
NGC 2623 & 083824.08 & +254516.40 & $18.3 \pm 0.2$ & $0830+241$ & $1.822 \pm 0.013$ \\
IRAS 08572+3915* & 090025.390 & +390354.40 & $3.18 \pm 0.3$ & $0927+390$ & $7.688 \pm 0.15$ \\
UGC 4881 & 091555.52 & +441957.5 & $3.5 \pm 0.5$ & $0920+446$ & $2.140 \pm 0.010$ \\
UGC 5101 & 093551.72 & +612111.29 & $18.0 \pm 1.0$ & $0958+655$ & $0.972 \pm 0.010$ \\
IRAS 10173+0828 & 102000.22 & +081334.5 & $2.9 \pm 0.4$ & $1041+061$ & $1.672 \pm 0.009$ \\
Arp 148 & 110353.95 & +405059.5 & $6.1 \pm 0.3$ & $1146+399$ & $1.330 \pm 0.012$ \\
UGC 6436 & 112545.15 & +144029.4 & $2.8 \pm 0.5$ & $1118+125$ & $1.238 \pm 0.016$ \\
IRAS 12112+0305 & 121346.1 & +024841.5 & $5.7 \pm 0.3$ & $1224+035$ & $0.788 \pm 0.006$ \\
UGC 8387 & 132035.34 & +340822.19 & $7.5 \pm 2.0$ & $1310+323$ & $0.963 \pm 0.042$ \\
UGC 8696 & 134442.11 & +555313.15 & $14.3 \pm 0.3$ & $1419+543$ & $0.935 \pm 0.023$ \\
IRAS 14348-1447 & 143738.30 & -150024.0 & $4.5 \pm 0.5$ & $1448-163$ & $0.376 \pm 0.006$ \\
IZw 107 & 151806.07 & +424445.19 & $3.6 \pm 0.3$ & $1521+436$ & $0.195 \pm 0.005$ \\
IRAS 15250+3609 & 152659.40 & +355837.53 & $4.2 \pm 0.3$ & $1521+436$ & $0.195 \pm 0.005$ \\
NGC 6286 & 165831.70 & +585614.50 & $12.2 \pm 1.5$ & $1638+573$ & $1.259 \pm 0.018$ \\
NGC 7469* & 230315.623 & +085226.39 & $17.50 \pm 0.50$ & $2320+052$ & $0.5661 \pm 0.004$ \\
IC 5298* & 231600.690 & +253324.20 & $3.86 \pm 0.18$ & $2321+275$ & $0.7178 \pm 0.009$ \\
Mrk 331* & 235126.802 & +203509.87 & $9.95 \pm 0.29$ & $2358+199$ & $0.2683 \pm 0.002$ \\
\hline
\end{tabular}

* Observations described in Prouton et al. (2004).
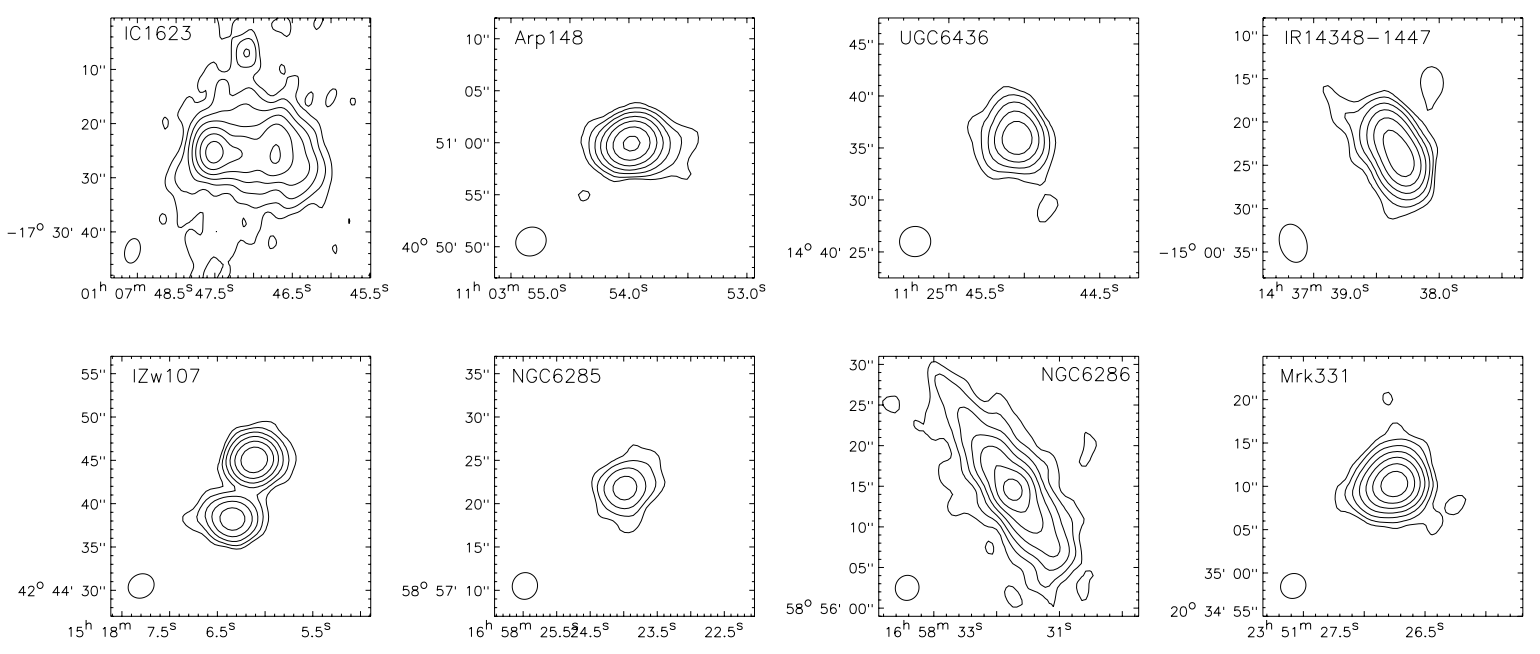

Fig. 2. Contour maps for the new $8.4 \mathrm{GHz}$ observations. The synthesised beam is shown in the bottom left of each panel. NGC 6285 is the companion of NGC 6286 and its radio map is included only for completeness.

suitable for the measurement of integrated fluxes ${ }^{1}$ were downloaded and reduced following standard procedures. Where the sources were of sufficient strength, a single iteration of selfcalibration was applied allowing for the correction of antenna phases only. Fluxes were measured by direct integration on the cleaned maps and are presented in Table 3. For UGC 08058 (Mrk 231) archival data were downloaded and reduced, despite the presence of measured fluxes in the literature, because of evidence that the radio emission from this source is variable. A significant fraction of its radio flux must therefore come from an

1 I.e., where the source extent was smaller than the largest angular scale which can be mapped by the VLA in the given configuration and frequency band.
AGN. The 5 fluxes listed in Table 3 were all measured on the same date. The maps of sources reduced from the VLA archive are shown in Fig. 3.

All calibration and mapping was carried using the NRAO Astonomical Image Processing System (AIPS). The radio fluxes for our sample galaxies (in the range $100 \mathrm{MHz}$ to $100 \mathrm{GHz}$ ) are listed in Table 1 in the Appendix, along with notes on selected sources.

\subsection{Data at other frequencies}

To make comparisons between our radio data and infrared data, we also collected $J, H$ and $K$-band fluxes from the 2MASS 
Table 2. 8.4 GHz fluxes for the newly observed objects.

\begin{tabular}{cccc}
\hline \hline Source & $\begin{array}{c}\text { Source flux } \\
(\mathrm{mJy})\end{array}$ & $\begin{array}{c}\text { Phase cal } \\
(\mathrm{J} 2000)\end{array}$ & $\begin{array}{c}\text { Phase cal flux } \\
(\mathrm{Jy})\end{array}$ \\
\hline IC 1623 & $54.6 \pm 3.0$ & $0118-216$ & $0.854 \pm 0.002$ \\
Arp 148 & $11.8 \pm 0.6$ & $1130+382$ & $1.338 \pm 0.005$ \\
UGC 6436 & $5.2 \pm 0.3$ & $1122+180$ & $0.639 \pm 0.003$ \\
IRAS 14348-1447 & $10.8 \pm 0.5$ & $1439-155$ & $0.509 \pm 0.003$ \\
IZw 107 & $14.3 \pm 0.7$ & $1500+478$ & $0.341 \pm 0.001$ \\
NGC 6286 & $31.5 \pm 1.6$ & $1638+573$ & $1.261 \pm 0.003$ \\
Mrk 331 & $22.3 \pm 1.0$ & $0010+174$ & $0.670 \pm 0.002$ \\
\hline
\end{tabular}

Extended Source Catalogue (Jarrett et al. 2000) and 12, 25, 60 and $100 \mu \mathrm{m}$ fluxes from the IRAS Faint Source Catalogue (Moshir et al. 1990).

In the collection of fluxes at other frequencies from the literature, care was taken to ensure that the fluxes were not confused or, in the case of interferometric radio data, underestimated due to high resolution. While most sources are compact and do not have other strong sources nearby, a small number of sources required corrections to some fluxes.

Confusion is most likely to be a problem for the IRAS fluxes due to the very low resolution. To try to quantify any possible problems with confusion in the absence of a higher resolution FIR survey, we used the NVSS and the FIR-radio correlation. In those cases where an unrelated radio source was within the IRAS beam, we reduced the IRAS flux by the fractional contribution of this source in the radio. Few sources were affected, but one source (IRAS 03359+1523) has its FIR emission confused with a source of similar strength. Because the error on a correction of this scale would render the fluxes unreliable, this source will not be considered in the model fitting described in Paper II. The ULIRGs for which the IRAS fluxes were reduced because of the presence of a nearby contaminating source were as follows: UGC 6436 (26\%), IRAS 12112+0305 (15\%), NGC 6286 (6\%), UGC $5101(8 \%)$.

The full list of fluxes for the sample galaxies from the nearinfrared to the radio will be presented in Paper II, where they are used to model the SEDs.

\section{Results}

\subsection{Radio spectral indices}

Before describing the observed radio spectra, we recall the expected, general form of the radio spectrum for a compact starburst, as outlined in Sect. 1. At frequencies, $v \sim 10 \mathrm{GHz}$, a steep synchrotron spectrum dominates, with $S_{v} \propto v^{\alpha}, \alpha \sim-0.8$. At both lower and higher frequencies the spectrum may be expected to flatten due to free-free absorption (low frequencies) and freefree emission (high frequencies).

In Fig. 4 we show the radio spectra for all our sources and Table 4 lists the radio spectral indices, $\alpha$, for the sample objects in several frequency ranges. Also shown are the FIR-radio flux ratios, $q=\log \left(F I R / 3.75 \times 10^{12} \mathrm{~Hz}\right) / S_{v}$, where $S_{v}$ is the radio flux in units of $\mathrm{W} \mathrm{m}^{-2} \mathrm{~Hz}^{-1}$ (Condon et al. 1991a). We use the notation, $q_{1.4}$, to refer to this quantity calculated at $1.4 \mathrm{GHz}$.

The mean value of $\alpha$ between 1.4 and $4.8 \mathrm{GHz}\left(\alpha_{4.8}^{1.4}\right.$ hereafter) is -0.521 . This steepens steadily towards higher frequencies, with $\alpha_{8.4}^{4.8}=-0.698, \alpha_{22.5}^{8.4}=-0.813$, and $\alpha_{22.5}^{15}=-0.842$ (although only 12 objects have values in this last range). This trend can also be seen in the lower-right panel of Fig. 4. At low frequencies, the flatter spectra are probably due to free-free absorption. However, the fact that the spectra steepen to frequencies as high as $22.5 \mathrm{GHz}$ is unexpected, since these sources should have very significant free-free emitting components, which would tend to flatten the spectra at high frequencies. Some sources even show spectral indices, $\alpha_{22.5}^{8.4}$, which are steeper than what is expected from pure synchrotron emission. The general trend, and these sources in particular, are further discussed in Sect. 5.3.

In Fig. 5 we show how the radio spectral index is related to $q$. There is a clear tendency for higher values of $q_{1.4}$ to be associated with radio spectral indices which are flatter, on average, from 1.4 to $22.5 \mathrm{GHz}$. There is also a clear correlation between $q_{1.4}$ and $\alpha_{4.8}^{1.4}$, with a much weaker correlation between $q_{1.4}$ and $\alpha_{8.4}^{4.8}$. On the other hand, $q_{8.4}$ is not correlated with either $\alpha_{4.8}^{1.4}$ or $\alpha_{8.4}^{4.8}$. This trend would be observed if the $1.4 \mathrm{GHz}$ radio fluxes were reduced by the effects of free-free absorption. In this case, both $q_{1.4}$ and $\alpha_{4.8}^{1.4}$ are affected. The $4.8 \mathrm{GHz}$ flux should be much less affected than the $1.4 \mathrm{GHz}$ flux because the free-free optical depth depends on frequency as $v^{-2.1}$. The $8.4 \mathrm{GHz}$ flux is free from the effects of absorption, and so no correlation is seen between $q_{8.4}$ and $\alpha_{4.8}^{1.4}$ or $\alpha_{8.4}^{4.8}$.

At high frequencies we see a correlation between $q_{8.4}$ and $\alpha_{22.5}^{8.4}$. The correlation seen in the lower right panel of Fig. 5 shows that the fractional contribution of free-free emission is larger in sources with higher values of $q_{8.4}$. Therefore, despite the fact that the average source shows a radio spectrum which steepens towards higher frequencies, flatter spectral indices tend to occur in sources with higher $q_{8.4}$. This is expected if the synchrotron emission is powered by SN explosions because of the delay between the formation of massive stars and the occurrence of the first SNe.

The trend for higher values of $q$ to be associated with flatter radio spectra over the whole range from 1.4 to $22.5 \mathrm{GHz}, \alpha_{22.5}^{1.4}$, will be used as a constraint on the starburst age in the model fitting of Paper II.

\subsection{Possible AGN contribution to the radio fluxes}

If there are ULIRGs in our sample that contain an AGN it is possible that this AGN contributes to the radio flux of the source. If the power-law index of the radio emission is different from that of the star formation we may expect to see a correlation between the radio spectral index and the near-infrared colours, which are sensitive to the presence of an AGN. In Fig. 6 we plot the radio spectral index against the near-infrared colour index $(J-K)$. There is no indication of a general correlation, which suggests that most sources do not contain a radio loud AGN. However, we do note that UGC 8058 (Mrk 231) which has the highest value of $(J-K)$ also has a very flat radio spectral index. As mentioned above, there is evidence that the AGN in this source is radio loud.

\subsection{Radio spectral indices and IRAS colour, $\left(f_{60} / f_{100}\right)$}

In Fig. 7 we plot the radio spectral indices against the IRAS colour $\left(f_{60} / f_{100}\right) \cdot \alpha_{4.8}^{1.4}$ shows a correlation with IRAS colour (in good agreement with the results of Sopp \& Alexander 1992) but this correlation almost disappears if $\alpha_{22.5}^{8.4}$ is considered instead. Given that warmer IRAS colours are accompanied by larger FIR fluxes (Young et al. 1989; Soifer et al. 1989) warmer IRAS colours imply higher rates of star formation. Therefore sources with warmer colours should have larger masses of ionised gas. However, the weakness of the correlation between $\left(f_{60} / f_{100}\right)$ and $\alpha_{22.5}^{8.4}$ suggests that this effect is not the main driver of the stronger correlation seen with $\alpha_{4.8}^{1.4}$. 
Table 3. Fluxes derived from archival VLA data.

\begin{tabular}{|c|c|c|c|c|c|c|c|c|}
\hline Source & $\begin{array}{c}\text { Frequency } \\
(\mathrm{GHz})\end{array}$ & $\begin{array}{c}\text { Flux } \\
(\mathrm{mJy})\end{array}$ & $\begin{array}{l}\text { Phase cal } \\
(\mathrm{J} 2000)\end{array}$ & $\begin{array}{c}\text { Phase cal flux } \\
\text { (Jy) }\end{array}$ & Program ID & Array & $\overline{\text { Date }}$ & $n$ \\
\hline NGC 34 & 4.8 & $28.4 \pm 0.3$ & $2358-103$ & $0.8080 \pm 0.0013$ & AM451 & $\bar{B}$ & 1994-Jul.-24 & 6 \\
\hline IIIZw 35 & 15 & $10.0 \pm 1$ & $0149+189$ & $0.3538 \pm 0.003$ & TYP04 & B & 2002-Sep.-06 & -1 \\
\hline NGC 1614 & 8.4 & $40.7 \pm 0.3$ & 0423-013 & $3.1178 \pm 0.004$ & AK331 & $\mathrm{C}$ & 1993-Aug.-03 & 6 \\
\hline IRAS 05189-2524 & 15 & $7.8 \pm 0.2$ & 0513-219 & $0.92 \pm 0.01^{*}$ & AC624 & $\mathrm{C}$ & 2003-Jan.-05 & 4 \\
\hline \multirow[t]{2}{*}{ IRAS $10173+0828$} & 4.8 & $5.88 \pm 0.2$ & $1150-003$ & $1.7056 \pm 0.004$ & AK184 & $\mathrm{CD}$ & 1988-Mar.-28 & 4 \\
\hline & 15 & $3.1 \pm 0.3$ & $1150-003$ & $0.9135 \pm 0.004$ & AK184 & $\mathrm{CD}$ & 1988-Mar.-28 & 1 \\
\hline \multirow[t]{2}{*}{ Arp 148} & 4.8 & $14.0 \pm 0.5$ & $1130+382$ & $0.7813 \pm 0.002$ & AH333 & A & 1989-Jan.-09 & 5 \\
\hline & 15 & $8.0 \pm 1$ & $1130+382$ & $0.6237 \pm 0.007$ & $\mathrm{AJ} 105$ & $\mathrm{C}$ & 1984-Мау-24 & 4 \\
\hline \multirow[t]{3}{*}{ Arp 299} & 8.4 & $243 \pm 8$ & $1219+484$ & $0.7873 \pm 0.003$ & AW641 & $\mathrm{C}$ & 2005-Aug.-20 & -1 \\
\hline & 15 & $160 \pm 3$ & $1219+484$ & $0.8489 \pm 0.012$ & AS568 & $\mathrm{C}$ & 1999-Jan.-21 & 2 \\
\hline & 22.5 & $74 \pm 2$ & $1128+594$ & $0.4509 \pm 0.004$ & AN095 & $\mathrm{C}$ & 2001-Aug.-25 & 2 \\
\hline \multirow[t]{2}{*}{ IRAS $12112+0305$} & 4.8 & $14.7 \pm 0.2$ & $1150-003$ & $1.7056 \pm 0.004$ & AK184 & $\mathrm{CD}$ & 1988-Mar.-28 & 3 \\
\hline & 15 & $5.7 \pm 0.2$ & $1150-003$ & $0.9135 \pm 0.004$ & AK184 & $\mathrm{CD}$ & 1988-Mar.-28 & 1 \\
\hline \multirow[t]{5}{*}{ UGC 08058} & 1.4 & $274 \pm 3$ & $1219+484$ & $0.5987 \pm 0.001$ & BU006Y & $\mathrm{B}$ & 1995-Nov.-17 & 1 \\
\hline & 4.8 & $265 \pm 1$ & $1219+484$ & $0.7885 \pm 0.002$ & BU006Y & B & 1995-Nov.-17 & 3 \\
\hline & 8.4 & $189 \pm 1$ & $1219+484$ & $0.9363 \pm 0.002$ & BU006Y & B & 1995-Nov.-17 & 4 \\
\hline & 15 & $146 \pm 1$ & $1219+484$ & $1.0916 \pm 0.005$ & BU006Y & B & 1995-Nov.-17 & 3 \\
\hline & 22.5 & $137 \pm 2$ & $1219+484$ & $1.1123 \pm 0.008$ & BU006Y & B & 1995-Nov.-17 & 0 \\
\hline UGC 8387 & 15 & $12 \pm 2$ & 3C286 & 3.45375 & AN35 & $\mathrm{C}$ & 1985-Aug.-30 & 0 \\
\hline \multirow[t]{2}{*}{ NGC 5256} & 8.4 & $24.1 \pm 1$ & $1417+461$ & $0.5880 \pm 0.003$ & AP233 & $\mathrm{C}$ & 1992-Apr.-28 & 4 \\
\hline & 15 & $21.0 \pm 1$ & $1349+536$ & $0.6003 \pm 0.006$ & AM198 & $\mathrm{CD}$ & 1987-Jan.-22 & 2 \\
\hline UGC 8696 & 15 & $29.5 \pm 1$ & $1349+536$ & $0.6003 \pm 0.006$ & AM198 & $\mathrm{CD}$ & 1987-Jan.-22 & 2 \\
\hline NGC 7469 & 8.4 & $50.2 \pm 0.6$ & $2320+052$ & $0.5897 \pm 0.05$ & AP233 & $\mathrm{C}$ & 1992-Apr.-28 & 3 \\
\hline
\end{tabular}

${ }^{*}$ No flux calibrator observed. Flux of 0513-219 set to this value using the VLA calibrator database. The last column, $n$, shows the scaling of the contours in Fig. 3.

The compactness of the regions is probably what causes the correlation in the upper panel of Fig. 7. If warmer IRAS colours were associated with more compact sources, then greater freefree absorption would be seen in warmer sources and a correlation between $\left(f_{60} / f_{100}\right)$ and $\alpha_{4.8}^{1.4}$ would result, even if the mass of ionised gas does not change with $\left(f_{60} / f_{100}\right)$. Source geometry is considered in more detail in Sect. 5.

\section{Discussion}

\subsection{The radio spectral index and the FIR-radio flux ratio, $q$}

In Fig. 4 there are several sources in which both a low and a high frequency spectral flattening are seen (e.g. IRAS 12112+0305). This flattening is due to free-free absorption at low frequencies and free-free emission at high frequencies. However, there are also sources which show evidence of free-free emission at high frequencies, with no sign of free-free absorption at low frequencies (e.g. CGCG436) and others that show signs of free-free absorption, with no sign of free-free emission (e.g. NGC 2623). The simplest explanation for these contrasting radio spectra is probably variations in the compactness of the emitting regions.

For a region of radius $r$ and density $N_{\mathrm{H}}$ maintained in ionisation equilibrium by a source of ionising radiation of fixed luminosity, the total ionisation/recombination rate, (4/3) $\pi r^{3} N_{\mathrm{H}}^{2} \alpha_{\mathrm{B}}$, where $\alpha_{\mathrm{B}}$ is the recombination coefficient, is constant (Osterbrock 1989). The free-free optical depth $\tau_{\mathrm{ff}} \propto$ $n_{\mathrm{e}}^{2} r$, for a density of ionised gas $n_{\mathrm{e}}$. Therefore, as long as $\tau_{\mathrm{ff}}$ remains below unity (certainly true at $22 \mathrm{GHz}$ ) the integrated free-free emission, $\propto \tau_{\mathrm{ff}} r^{2}$, from a region with fixed ionising luminosity, is independent of the source size.

However, where this gas absorbs a luminosity source, the absorption is proportional to $\mathrm{e}^{-\tau_{\mathrm{ff}}}$ (foreground screen) and the dependence of $\tau_{\mathrm{ff}}$ on source size means that the compactness of a region very strongly affects the amount of absorption. For a given ionising luminosity and synchrotron luminosity, an extended region may show no evidence of free-free absorption at $1.4 \mathrm{GHz}$, whereas a more compact region will. This effect is probably behind the the diverse spectral shapes of Fig. 4 and is also important in understanding Fig. 5.

In considering the relation between the radio spectral indices at various frequencies and $q$, shown in Fig. 5, it is useful to keep in mind how each are related to the star formation rate. Both the FIR and the integrated free-free emission are expected to be proportional to the current star formation rate. The synchrotron emission is also proportional to the star formation rate, but as the emission is delayed by the main sequence lifetime of massive stars, it measures the star formation rate $\sim 10^{7}$ years prior to the current epoch. Therefore, in objects characterised by bursts of star formation, such as those in our sample, we expect both $\alpha$ and $q$ to be a function of the age of the starburst. Values of $q$ are higher where the current star formation rate is greater than that $\sim 10^{7}$ years ago, and lower where the star formation rate has declined. In this scenario, $q$ is a measure of the "age" of a starburst, with higher values associated with younger objects.

In Fig. 5, as well as the correlations between $q_{1.4}$ and $\alpha_{4.8}^{1.4}$, and between $q_{8.4}$ and $\alpha_{22.5}^{8.4}$, we note the lack of a correlation between $q_{8.4}$ and $\alpha_{4.8}^{1.4}$. If large values of $q_{8.4}$ are due to a delay in the production of synchrotron emission relative to that of thermal dust and free-free emission, as the correlation between $q_{8.4}$ and $\alpha_{22.5}^{8.4}$ suggests, then the lack of correlation between $q_{8.4}$ and $\alpha_{4.8}^{1.4}$ implies that the age of the starburst is not the only effect.

Because source compactness strongly affects the amount of free-free absorption, but does not affect the free-free emission (for $\tau_{\mathrm{ff}} \ll 1$ ) the presence of free-free emission at $22.5 \mathrm{GHz}$ does not imply the presence of free-free absorption at $1.4 \mathrm{GHz}$. Therefore, although the youngest sources (highest $q_{8.4}$ values) have larger ionised gas fractions, there is a range of source 
M. S. Clemens et al.: Modeling the spectral energy distribution of ULIRGs. I.

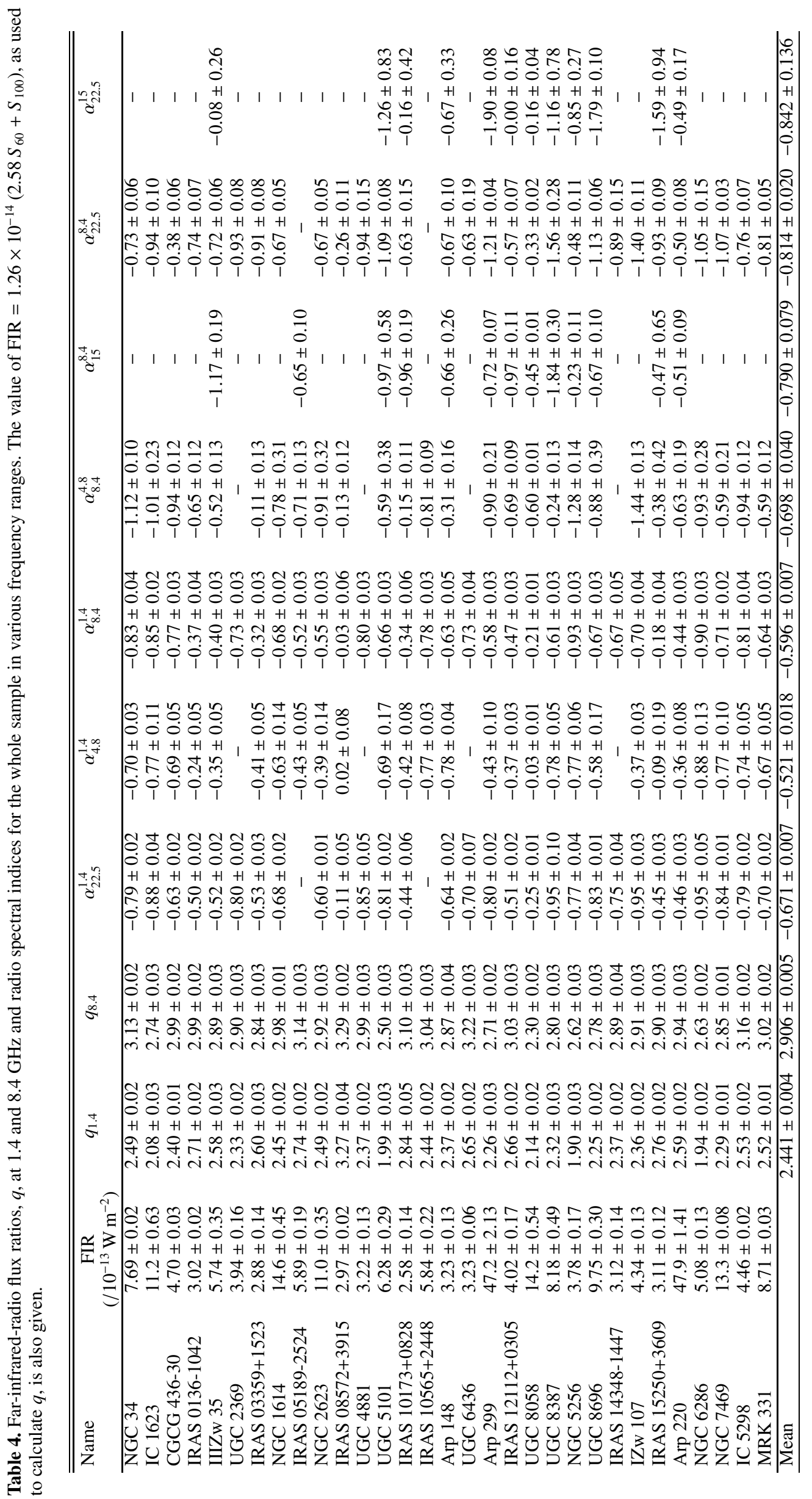



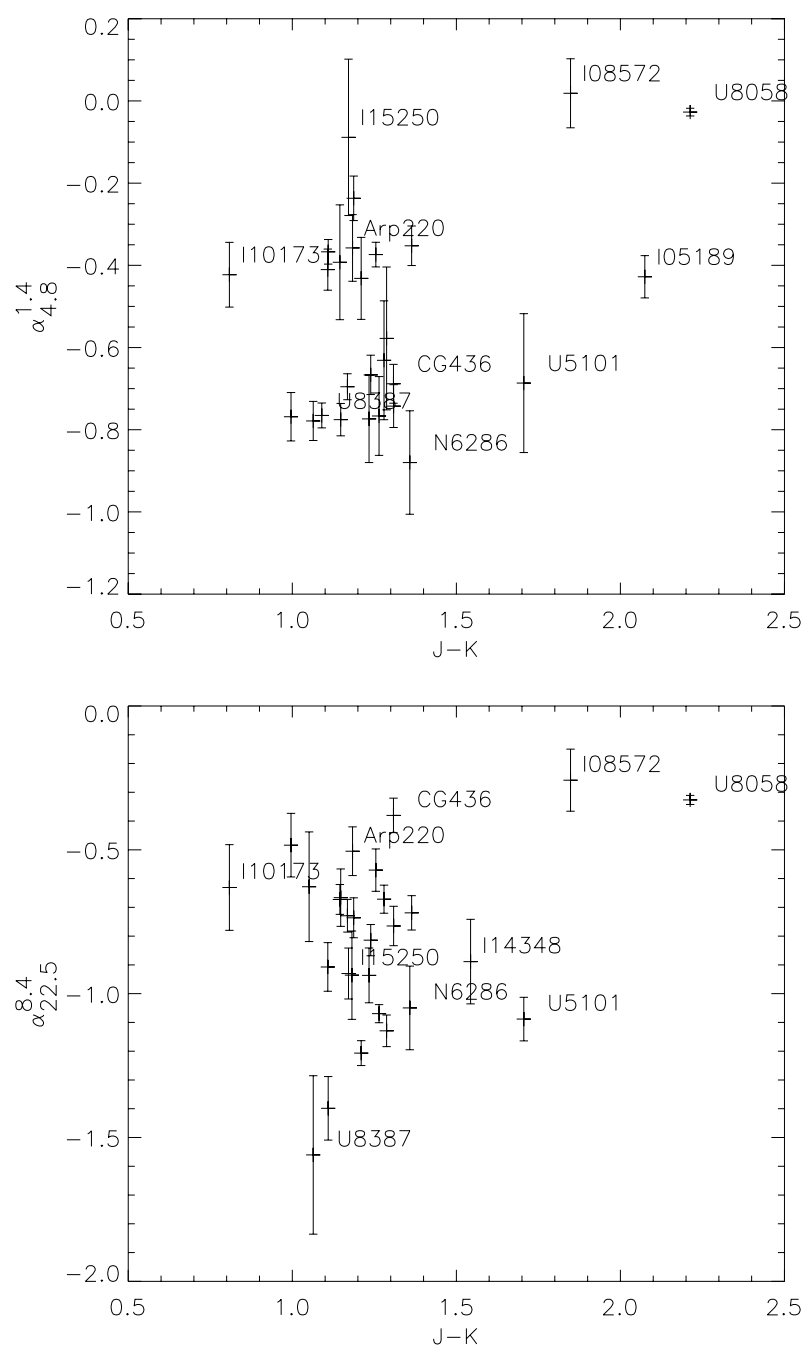

Fig. 6. The relation between the near-infrared colour $J-K$ and the radio spectral index between 1.4 and $4.8 \mathrm{GHz}$ (top) and 8.4 and $22.5 \mathrm{GHz}$ (bottom). Objects at the extremes of the distributions are labelled.

compactness for any given age. This causes the lack of correlation between $q_{8.4}$ and $\alpha_{4.8}^{1.4}$.

\subsection{Low frequency radio spectral index and the FIR colour}

As seen in Fig. 7 the low frequency radio spectral index $\alpha_{4.8}^{1.4}$ is correlated with the FIR flux ratio $f_{60} / f_{100}$. Warmer FIR colours are therefore associated with more free-free absorption. However, we recall that younger sources do not show more freefree absorption (Fig. 5, $\alpha_{4.8}^{1.4}$ vs. $q_{8.4}$ ). Together, these imply that the warmest dust is not found in the youngest sources, as has been suggested previously (Vega et al. 2005). If source compactness has an important role to play in determining the level of free-free absorption at low radio frequencies, then the most compact are those in which the dust is warmest.

\subsection{High frequency radio spectral indices}

A small number of ULIRGs in our sample show surprisingly steep spectral indices at high frequencies. UGC 5101, Arp 299, UGC 8387, UGC 8696, IZw 107, NGC 6286 and NGC 7469 all have radio spectral indices between 8.4 and $22.5 \mathrm{GHz}$ steeper than $\alpha=-1$. There appears to be either a deficit in the observed
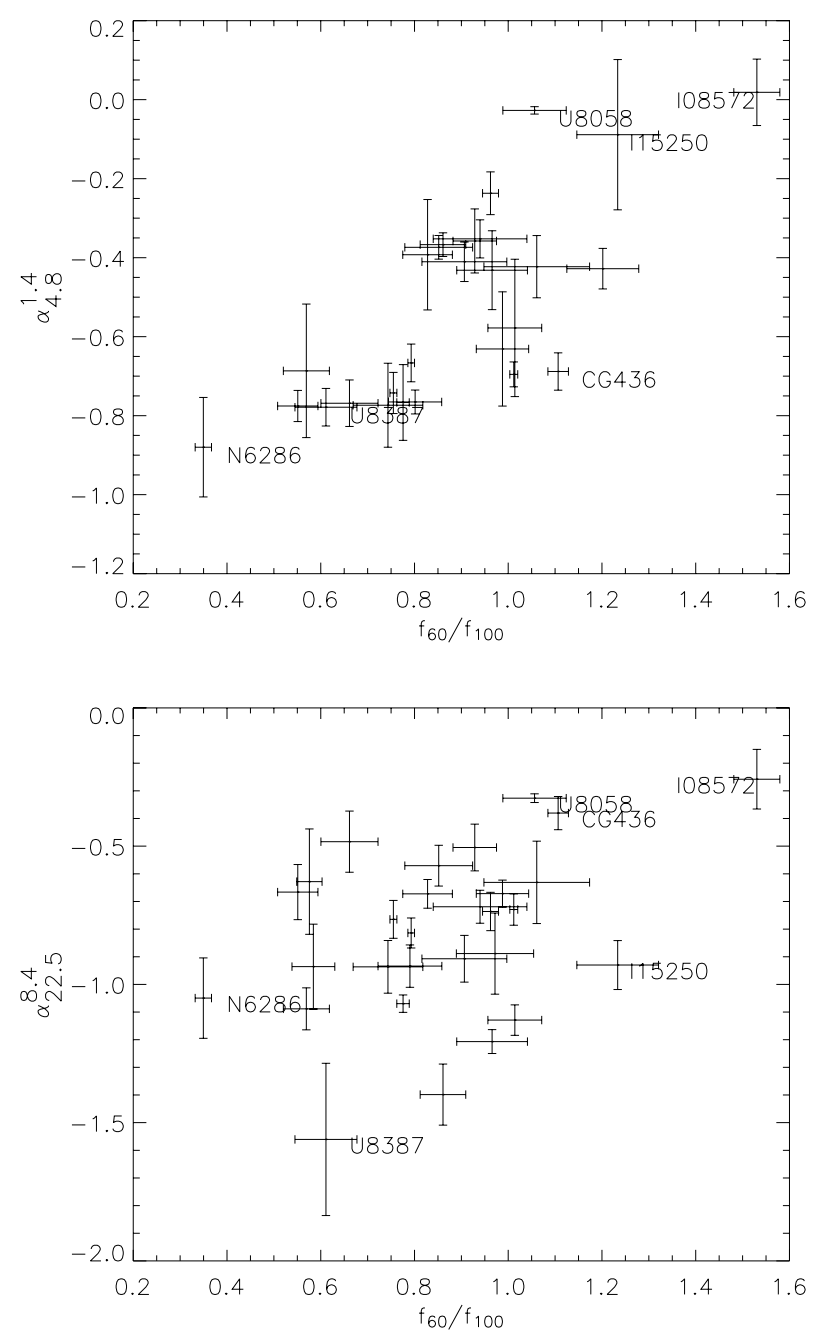

Fig. 7. Top: correlation between the radio spectral index between 1.4 and $4.8 \mathrm{GHz}$ and IRAS 60/100 micron flux ratio. Bottom: same plot for the $8.4-22.5 \mathrm{GHz}$ spectral index. Objects at the extremes of the distributions are labelled. Note that that UGC 8058 may have a radio loud AGN.

flux at $22 \mathrm{GHz}$ or a spectrum that is also very steep at lower frequencies (UGC 5101, IZw 107, NGC 6286). In the case of IZw 107 the radio spectrum has a spectral index of $\alpha=-1.4$ from 4.8 to $22.5 \mathrm{GHz}^{2}$. In those cases where the radio spectra show a steepening towards higher frequencies the spectrum appears to show a break around $15 \mathrm{GHz}$.

This is contrary to what we expect in sources with a large mass of free-free emitting gas. The radio spectrum should flatten at high frequencies due to the increasing importance of the thermal emission $\left(S_{v} \propto v^{-0.1}\right)$. Although it is conceivable that the mass of free-free emitting gas is reduced by the absorption of ionising photons by dust, this effect alone cannot produce an observed radio spectrum that steepens towards higher frequencies.

A steepening of the radio spectrum towards higher frequencies is expected in the presence of "aging" of the relativistic electron population (Condon 1992). Because synchrotron (and inverse Compton) losses are greater at higher frequencies $\left(\tau_{\text {sync }} \propto v^{-0.5}\right)$ higher energy electrons emit synchrotron photons for shorter times than those of lower energies. For an instantaneous injection of relativistic electrons the radio spectrum

2 Note, however, that a steeper power-law fit to the high frequency radio data would not fit the $1.4 \mathrm{GHz}$ and $408 \mathrm{MHz}$ data points. 
therefore steepens with increasing frequency. The "break" frequency which parameterises this steepening moves to lower frequencies at later times after the injection. Spectral aging effects were considered by Colbert et al. (1994) to explain the steep radio spectral index in the non-nuclear regions of NGC 6240.

In ULIRGs the electron lifetimes are very short because in a high radiation energy density environment, inverse Compton losses would remove all high energy electrons before they could emit synchrotron photons. The observation of synchrotron emission in such sources therefore shows that they must have strong B-fields and short electron lifetimes due to synchrotron losses. Electron lifetimes in the $\mathrm{GHz}$ region are shorter not only than a starburst timescale $\left(10^{7}-10^{8} \mathrm{yr}\right)$ but also than the lifetime of the most massive stars $\left(\sim 5 \times 10^{6} \mathrm{yr}\right)$. Therefore the production of relativistic electrons cannot be considered instantaneous, but is rather close to a continuous injection approximation.

Under the assumption of continuous injection of relativistic electrons, the radio spectrum can be considered as the superposition of several instantaneously injected populations, each with a different age, and therefore each with a different "break" frequency. The spectrum which results from the summation of all these components is straight. If the asymptotic change in spectral index for a single component of spectral index, $\alpha$, is $\Delta \alpha=-0.5$ (such as in the "dynamical halo" model of Lerche $\&$ schlickeiser 1981) then the resulting spectrum has a spectral index $\alpha_{\text {aged }}=\alpha-0.5$. The radio spectra of all ULIRGs should therefore be steepened by the effects of spectral aging, but we do not expect to detect a down-turn in the spectra towards high frequencies if continuous injection is a good approximation. We note however, that because the spectral break for an aging, instantaneously injected electron population spends little time in the $\sim 10 \mathrm{GHz}$ range (where we observe the putative breaks) the form of the time-averaged spectrum is rather sensitive to stochastic effects in the recent past. Such effects will be important where the SN rate shows variations on timescales of the order of the electron lifetime.

Qualitatively, such a stochastic effect could be caused by a radio hypernova, which might have an order of magnitude greater flux than a typical SN. However, it seems very unlikely that a single such source could effect the integrated radio spectrum of an entire ULIRG. The compact sources mapped in Arp 220 by Lonsdale et al. (2006) account for only $6 \%$ of the total flux at $18 \mathrm{~cm}$.

Lisenfeld et al. (2004) considered various explanations for the high frequency turn over seen in the synchrotron spectrum of the starburst dwarf galaxy NGC 1569, once the contribution of thermal emission had been subtracted. They concluded that a sharp turn over could only be achieved by either a rapid temporal variation of the star formation rate or through the preferential escape of low-energy electrons from the galaxy's disc. In NGC 1569 a starburst that started abruptly $10^{7}$ yr ago was found to reproduce the observed break in the synchrotron spectrum. The escape of low energy electrons from the galaxy can produce a break if convection can transport the electrons out of the disc during their radiative lifetime. As the lower energy electrons suffer fewer synchrotron losses, and therefore emit for longer than high-energy electrons, they can in principle be lost preferentially and cause a break in the spectrum. Lisenfeld et al. (2004) found that a convective wind velocity of $150 \mathrm{~km} \mathrm{~s}^{-1}$ can reproduce the spectral break in NGC 1569. However, the lifetimes of electrons against radiative losses in ULIRGs should be considerably shorter than in NGC 1569. Under the assumption of energy equipartition between the radiation and magnetic fields, Condon et al. (1991a) estimate electron lifetimes of $\sim 10^{4} \mathrm{yr}$ for electrons radiating at $8.4 \mathrm{GHz}$ in the same sample of ULIRGs from which ours were selected, while that in NGC 1569 was estimated to be $5 \times 10^{6} \mathrm{yr}$ by Lisenfeld et al. Because the supernova rate cannot change significantly over timescales of $10^{4} \mathrm{yr}^{3}$ and a convective wind could not remove electrons from the sources over similar timescales, none of these mechanisms seem a plausible explanation for a high frequency spectral break in the present sample of ULIRGs.

Although there is no doubt that some sources have steep spectral indices from 1 to $20 \mathrm{GHz}$, in the majority of cases where a turn-over is seen it is due only to the $22.5 \mathrm{GHz}$ data point (Arp 299, UGC 8696, IRAS 15250+3609). We would require data at a higher frequency (e.g. $43 \mathrm{GHz}$ ) for these sources to be sure of the spectral bend and warrant a more in-depth discussion.

\section{Conclusions}

We have presented new high frequency radio data for a sample of ULIRGs, which, together with re-reduced archival data and fluxes taken from the literature forms a sample of 31 ULIRGS with well-sampled radio spectra. All but one source have a measured flux at 22.5 GHz. Every effort has been made to ensure that the fluxes are reliable measures of the integrated radio emission. This means that the possibility of missing flux in high-resolution interferometer data and of confusing sources in low resolution data has been considered. We find the following,

- Few sources have straight power-law slopes. Although the individual spectra show a variety of spectral bends the trend of the mean spectrum is a steepening from $1.4 \mathrm{GHz}$ to $22.5 \mathrm{GHz}$. Above and below these frequencies data are too sparse to define a meaningful average.

- The low frequency radio spectral index between 1.4 and $4.8 \mathrm{GHz}$ is correlated with the FIR-radio flux density ratio $\mathrm{q}$ calculated at $1.4 \mathrm{GHz}$. This correlation is due to free-free absorption of low frequency synchrotron photons by ionised gas.

- The high frequency radio spectral index between 8.4 and $22.5 \mathrm{GHz}$ is correlated with the FIR-radio flux density ratio $\mathrm{q}$, calculated at $8.4 \mathrm{GHz}$. This correlation is due to a higher fractional contribution from free-free emission in sources with higher values of $q_{8.4}$. These are the younger sources.

- Given the above correlations the lack of a correlation between the radio spectral index between 1.4 and $4.8 \mathrm{GHz}$ and $q_{8.4}$ is evidence that the compactness of the emitting regions plays an important role in defining the slope of the low frequency radio spectrum.

- The fact that the low frequency radio spectral index $\alpha_{4.8}^{1.4}$ is correlated with the FIR flux ratio $f_{60} / f_{100}$ (while $\alpha_{22.5}^{8.4}$ is not) suggests that the ULIRGs in which the emitting regions are most compact are those with the warmest IRAS $f_{60} / f_{100}$ colours.

If the FIR-to-radio flux ratio measured at $8.4 \mathrm{GHz}, q_{8.4}$, is a measure of the age of the star formation in ULIRGs (larger values being "younger") we find that the youngest objects have a larger contribution to their radio fluxes from free-free emission, but do not have the most free-free absorption. The sources with

\footnotetext{
3 Type $1 \mathrm{~b}$ and type II SNe are thought to be the source of the synchrotron emission in ULIRGs, and occur by the core-collapse of stars more massive than $8 M_{\odot}$. The difference in lifetime between a star of $8 M_{\odot}$ and a star of $100 M_{\odot}$ is $\sim 5 \times 10^{7} \mathrm{yr}$. Therefore, for a normal initial mass function, even if the stars formed instantaneously, the SN rate cannot show large variations on timescales as short as $10^{4} \mathrm{yr}$.
} 
most free-free absorption are instead those in which the emitting regions are more compact. Although these have the warmest FIR colours, they are not necessarily the youngest sources.

Observations at radio frequencies below $1.4 \mathrm{GHz}$ will be a very effective way of quantifying the effects of free-free absorption in these sources.

In Paper II these radio spectra, together with data from the radio to the near-infrared, will be used to constrain new models which reproduce the SED of compact starbursts with a possible AGN contribution.

Acknowledgements. M.C. acknowledges the the support of an INAF research fellowship. O.V. acknowledges the support of the INAOE and the Mexican CONACYT projects $36547 \mathrm{E}$ and 39714 F. A.B. acknowledges the warm hospitality of INAOE. A.B., G.L.G., and L.S. acknowledge partial funding by the European Community by means of the Maria Curie contract MRTN-CT-2004-503929, "MAGPOP". The National Radio Astronomy Observatory is a facility of the National Science Foundation operated under cooperative agreement by Associated Universities, Inc. This publication makes use of data products from the Two Micron All Sky Survey, which is a joint project of the University of Massachusetts and the Infrared Processing and Analysis Center/California Institute of Technology, funded by the National Aeronautics and Space Administration and the National Science Foundation. This research has made use of the NASA/IPAC Extragalactic Database (NED) which is operated by the Jet Propulsion Laboratory, California Institute of Technology, under contract with the National Aeronautics and Space Administration.

\section{References}

Alonso-Herrero, A., Rieke, G. H., Rieke, M. J., \& Scoville, N. Z. 2000, ApJ, 532,845

Alonso-Herrero, A., Rieke, G. H., Rieke, M. J., \& Scoville, N. Z. 2002, AJ, 124, 166

Anantharamaiah, K. R., Viallefond, F., Mohan, N. R., Goss, W. M., \& Zhao, J. H. 2000, ApJ, 537, 613

Baan, W. A., \& Klöckner, H.-R. 2006, A\&A, 449, 559

Bressan, A., Silva, L., \& Granato, G. L. 2002, A\&A, 392, 377

Clemens, M. S., \& Alexander, P. 2004, MNRAS, 350, 66

Colbert, E. J. M., Wilson, A. S., \& Bland-Hawthorn, J. 1994, ApJ, 436, 89

Condon J. J. 1992, ARA\&A, 30, 575

Condon, J. J., Condon, M. A., Broderick, J. J., \& Davis, M. M. 1983, AJ, 88, 20

Condon, J. J., Huang, Z.-P., Yin, Q. F., \& Thuan, T. X. 1991a, ApJ, 378, 65

Condon, J. J., Frayer, D. T., \& Broderick, J. J. 1991b, AJ, 101, 362
Condon, J. J., Cotton, W. D., Greisen, E. W., et al. 1998, AJ, 115, 1693

Crawford, T., Marr, J., Partridge, B., \& Strauss, M. A. 1996, ApJ, 460, 225

Douglas, J. N., Bash, F. N., Bozyan, F. A., Torrence, G. W., \& Wolfe, C. 1996 , AJ, 111, 1945

Dressel, L. L., \& Condon, J. J. 1978, ApJS, 36, 53

Ficarra, A., Grueff, G., \& Tomassetti, G. 1985, A\&AS, 59, 255

Flores, H., Hammer, F., Elbaz, D., et al. 2004, A\&A, 415, 885

Gao, Y., \& Solomon, P. M. 2004, ApJS, 152, 63

Goldader, J. D., Joseph, R. D., Doyon, R., \& Sanders, D. B. 1995, ApJ, 444, 97

Goldader, J. D., Joseph, R. D., Doyon, R., \& Sanders, D. B. 1997, ApJ, 474, 104 Gregory, P. C., \& Condon, J. J. 1991, ApJS, 75, 1011

Griffith, M. R., Wright, A. E., Burke, B. F., \& Ekers, R. D. 1994, ApJS, 90, 179

Griffith, M. R., Wright, A. E., Burke, B. F., \& Ekers, R. D. 1995, ApJS, 97, 347

Imanishi, M., Nakanishi, K., \& Kohno, K. 2006, AJ, 131, 2888

Iono, D., Ho, P. T. P., Yun, M. S., et al. 2004, ApJ, 616, L63

Jarrett, T. H., Chester, T., Cutri, R., et al. 2000, AJ, 119, 2498

Lerche, I., \& Schlickeiser, R. 1981, ApL, 22, 31

Lisenfeld, U., Wilding, T. W., Pooley, G. G., \& Alexander, P. 2004, MNRAS, 349,1335

Lonsdale, C. J., Diamond, P. J., Thrall, H., Smith, H. E., \& Lonsdale, C. J. 2006, ApJ, 647, 185

Moshir, M., et al. 1990, IRAS Faint Source Catalogue, version 2.0

Murphy, T. W., Jr., Soifer, B. T., Matthews, K., Armus, L., \& Kiger, J. R. 2001, AJ, 121, 97

Nagar, N. M., Wilson, A. S., Falcke, H., Veilleux, S., \& Maiolino, R. 2003, A\&A, 409, 115

Poggianti, B. M., Bressan, A., \& Franceschini, A. 2001, ApJ, 550, 195

Prouton, O. R., Bressan, A., Clemens, M., et al. 2004, A\&A, 421, 115

Rodríguez-Rico, C. A., Goss, W. M., Viallefond, F., et al. 2005, ApJ, 633, 198

Rush, B., Malkan, M. A., \& Edelson, R. A. 1996, ApJ, 473, 130

Silva, L., Granato, G. L., Bressan, A., \& Danese, L. 1998, ApJ, 509, 103

Smith, H. E., Lonsdale, C. J., Lonsdale, C. J., \& Diamond, P. J. 1998, ApJ, 493, L17

Smith, H. E., Lonsdale, C. J., \& Lonsdale, C. J. 1998, ApJ, 492, 137

Soifer, B. T., Boehmer, L., Neugebauer, G., \& Sanders, D. B. 1989, AJ, 98, 766

Sopp, H., \& Alexander, P. 1992, MNRAS, 259, 425

Sopp, H., Alexander, P., \& Riley, J. 1990, MNRAS, 246, 143

Sopp, H. M., \& Alexander, P. 1991, MNRAS, 251, 112

Sramek, R. A., \& Tovmassian, H. M. 1976, ApJ, 207, 725

Valdés, J. R., Berta, S., Bressan, A., et al. 2005, A\&A, 434, 149

Vega, O., Silva, L., Panuzzo, P., et al. 2005, MNRAS, 364, 1286

Vega, O., Clemens, M. S., Bressan, A., et al. 2007, A\&A, submitted (Paper II)

Young, J. S., Xie, S., Kenney, J. D. P., \& Rice, W. L. 1989, ApJS, 70, 699

Yun, M. S., Scoville, N. Z., \& Knop, R. A. 1994, ApJ, 430, L109

Zhao, J.-H., Anantharamaiah, K. R., Goss, W. M., \& Viallefond, F. 1996, ApJ, 472,54 
M. S. Clemens et al.: Modeling the spectral energy distribution of ULIRGs. I., Online Material $p 1$

\section{Online Material}


M. S. Clemens et al.: Modeling the spectral energy distribution of ULIRGs. I., Online Material $p 2$
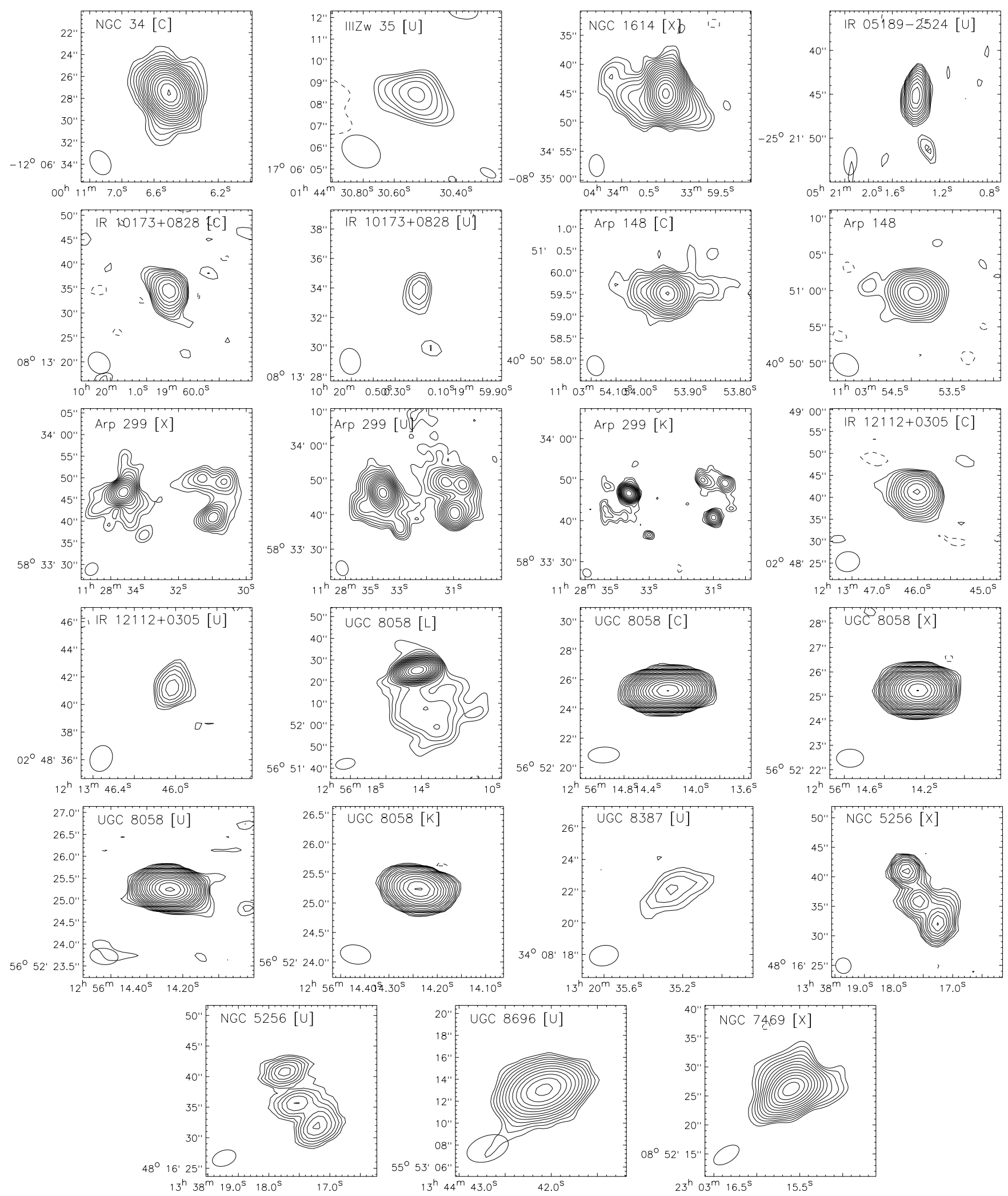

Fig. 3. Contour maps for the archival VLA data. In brackets after the name of the source is the name of the radio frequency band; $L(1.4 \mathrm{GHz})$, $C(4.8 \mathrm{GHz}), X(8.4 \mathrm{GHz}), U(15 \mathrm{GHz}), K(22.5 \mathrm{GHz})$. Contour levels are $2^{-n x_{i} / 2}$ where $x=0,1,2, \ldots$ The dotted contour has the same absolute value as the first contour but is negative. The value of $n$ for each contour map is included in Table 3 . The synthesised beam is shown at the bottom left of each panel. 
M. S. Clemens et al.: Modeling the spectral energy distribution of ULIRGs. I., Online Material p 3

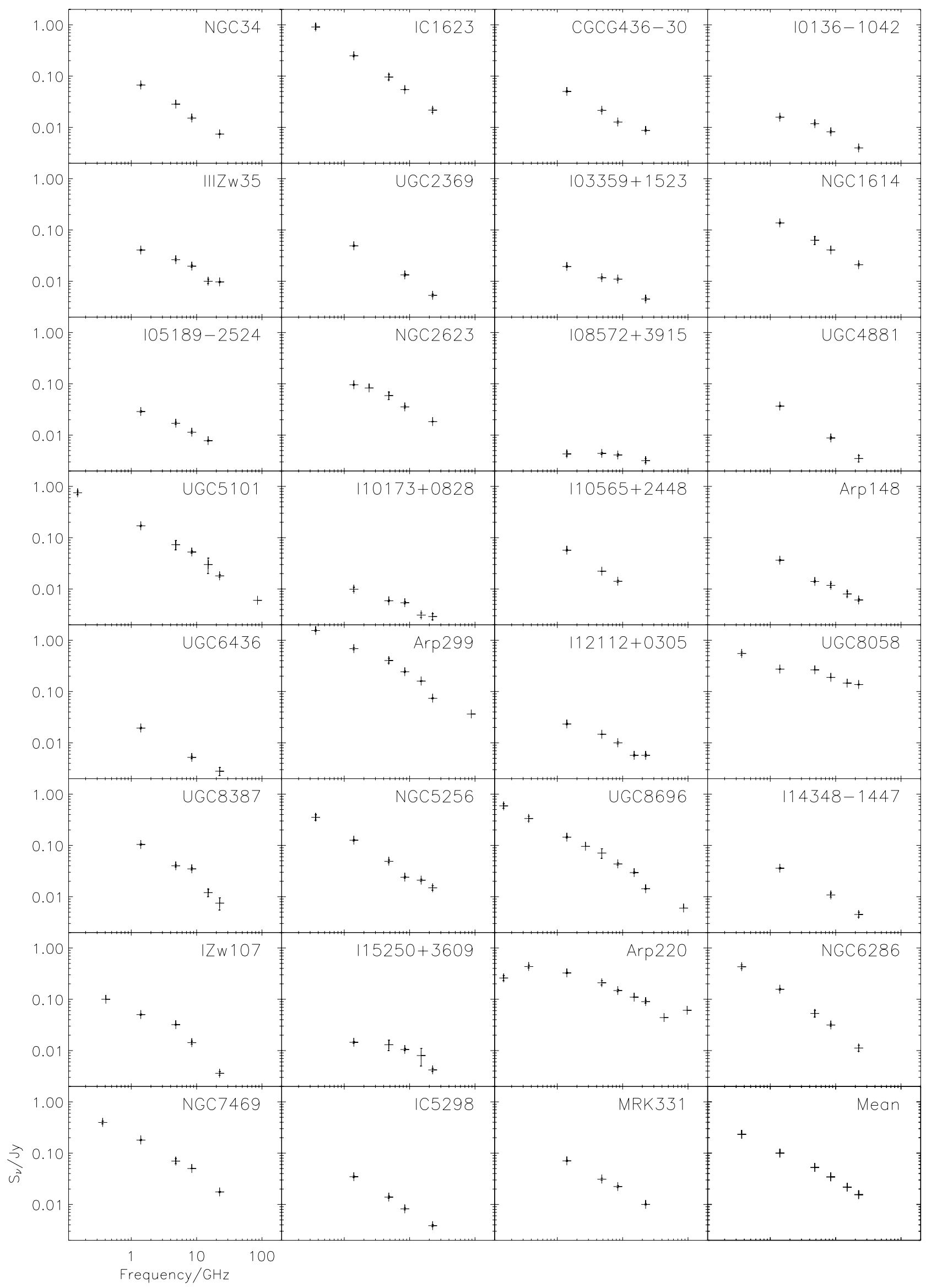

Fig. 4. The radio spectra of the sample galaxies from $100 \mathrm{MHz}$ to $100 \mathrm{GHz}$. The lower left panel shows the mean spectrum of all the sources scaled so that the $1.4 \mathrm{GHz}$ flux is $0.1 \mathrm{Jy}$. 
M. S. Clemens et al.: Modeling the spectral energy distribution of ULIRGs. I., Online Material p 4
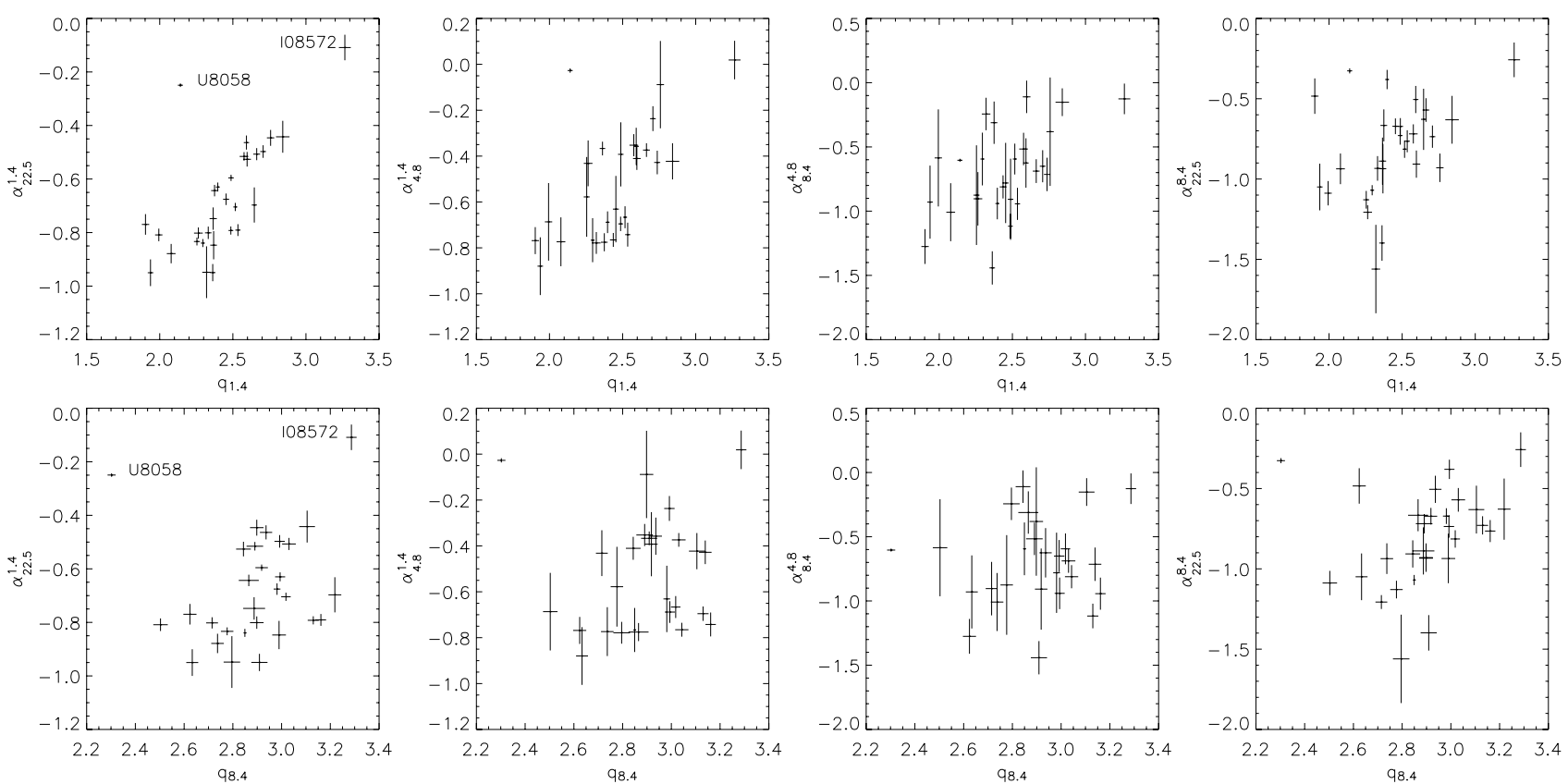

Fig. 5. Relationship between radio spectral index at various frequencies and the logarithmic FIR-radio flux density ratio $q$, defined as $q=$ $\log \left(F I R / 3.75 \times 10^{12} \mathrm{~Hz}\right) / S$, where $S$ is the radio flux in units of $\mathrm{W} \mathrm{m}^{-2} \mathrm{~Hz}^{-1}$. The top panels show the radio spectral index as a function of $q$ measured at $1.4 \mathrm{GHz}$ and the bottom panels show the radio spectral indices as a function of $q$ measured at $8.4 \mathrm{GHz}$. The left-most panels show the radio spectral index over the whole range from 1.4 to $22.5 \mathrm{GHz}$ while the remainder show the spectral indices over a more restricted range. 


\section{Appendix A: Individual objects}

Here we briefly describe aspects of the radio emission relevant to the derivation of integrated fluxes.

\section{IC 1623}

The $22.5 \mathrm{GHz}$ emission shows an unresolved peak at the position of the eastern nucleus with a clear extension towards the west. The distribution of emission is very similar to that of the molecular gas (Iono et al. 2004; Yun et al. 1994).

\section{IIIZw 35}

The radio emission originates from the more northern of the 2 optical nuclei.

\section{IRAS 03359+1523}

1 .'5 to the south of this object there is a $15.4 \mathrm{mJy}$ radio source. IRAS $03359+1523$ itself emits only $19.4 \mathrm{mJy}$ at $1.4 \mathrm{GHz}$. Rather than reducing the IRAS fluxes by nearly a factor of two we choose to exclude this object from the analysis of Paper II.

\section{UGC 4881}

We detect two sources in this object at the same locations as those revealed in the $8.4 \mathrm{GHz}$ observations of Condon et al. (1991a). The more eastern component is the brighter and accounts for $\sim 70 \%$ of the total flux detected, which is similar to the flux ratio at $8.4 \mathrm{GHz}$.

\section{UGC 5101}

A $12.3 \mathrm{mJy}$ radio source lies $1^{\prime}$ to the north-east. IRAS fluxes reduced by $8 \%$.

\section{IRAS 10173+0828}

The sub-arcsecond 15 and $22.5 \mathrm{GHz}$ observations of Smith et al. (1998) show an unresolved nuclear source.

\section{UGC 6436}

The NVSS shows that a companion galaxy, IC $2810 \mathrm{~b}$, located 1.2 to the south-east has a flux of $7.7 \mathrm{mJy}$ compared to the 19.4 mJy of UGC 6436. This would be confused at IRAS resolutions and so the IRAS fluxes have been reduced by $26 \%$. No other observations were at so low a resolution as to make contamination from this source a problem.

\section{Arp 299}

A close interacting pair consisting of NGC 3690 and IC 694.

\section{IRAS 12112+0305}

A source $2: 4$ to the south-east has a $1.4 \mathrm{GHz}$ flux of $5.1 \mathrm{mJy}$ compared to the $23.3 \mathrm{mJy}$ emitted by IRAS $12112+0305$. IRAS fluxes reduced by $15 \%$.

\section{UGC 8387}

Our $22.5 \mathrm{GHz}$ map shows an unresolved source but the higher resolution $8.4 \mathrm{GHz}$ radio continuum map of Condon et al. (1991a) shows an elongated structure with an extent of $\sim 4^{\prime \prime}$. Clemens \& Alexander (2004) find that free-free absorption flattens the radio spectral index towards the centre of the source showing there to be a large region of dense ionised gas.

The $22.5 \mathrm{GHz}$ flux for this source may be incorrect. The calibrator flux for this source was only about half that reported in the VLA calibrator database.

\section{IZw 107}

Our $8.4 \mathrm{GHz}$ map shows that the extended emission seen at $22.5 \mathrm{GHz}$ is due to the presence of 2 sources separated by $8^{\prime \prime}$.

\section{Arp 220}

Smith et al. (1998) resolved the compact radio emission into separate supernova remnants and subsequent, high sensitivity VLBI monitoring of both nuclei has obtained a direct estimate of the SN rate (Lonsdale et al. 2006) of $4 \pm 2 \mathrm{yr}^{-1}$. The implied star formation rate is sufficient to supply the bolometric luminosity of the system.

\section{NGC 6286}

The radio structure at 22 and $8 \mathrm{GHz}$ is elongated in the same sense as the optical, edge-on disc. A weak source (NGC 6285) is present 1.5 to the north-west and this is detected in our $8.4 \mathrm{GHz}$ data but is undetected in our $22.5 \mathrm{GHz}$ map. The IRAS fluxes were reduced by $6 \%$ to remove the probable contribution of NGC 6285.

Mrk 331

See Prouton et al. (2004). 
M. S. Clemens et al.: Modeling the spectral energy distribution of ULIRGs. I., Online Material p 6

Table A.1. Radio fluxes.

\begin{tabular}{|c|c|c|c|}
\hline Source & $\begin{array}{c}\text { Frequency } \\
(\mathrm{GHz})\end{array}$ & $\begin{array}{c}\text { Flux } \\
(\mathrm{mJy})\end{array}$ & $\overline{\text { Ref. }}$ \\
\hline \multirow[t]{4}{*}{ NGC 34} & 1.4 & $66.9 \pm 2.5$ & $\mathrm{~d}$ \\
\hline & 4.8 & $28.4 \pm 0.3$ & $\mathrm{c}$ \\
\hline & 8.4 & $15.2 \pm 0.8$ & $\mathrm{e}$ \\
\hline & 22.5 & $7.41 \pm 0.14$ & $\mathrm{~b}$ \\
\hline \multirow[t]{5}{*}{ IC 1623} & 0.365 & $906 \pm 83$ & $\mathrm{v}$ \\
\hline & 1.4 & $249 \pm 9.8$ & $\mathrm{~d}$ \\
\hline & 4.8 & $96 \pm 12$ & $\mathrm{i}$ \\
\hline & 8.4 & $54.6 \pm 1$ & a \\
\hline & 22.5 & $21.7 \pm 2$ & $\mathrm{a}$ \\
\hline \multirow[t]{4}{*}{ CGCG436-30 } & 1.4 & $49.8 \pm 1.5$ & d \\
\hline & 4.8 & 21.5 & g \\
\hline & 8.4 & $12.7 \pm 0.6$ & $\mathrm{e}$ \\
\hline & 22.5 & $8.73 \pm 0.31$ & b \\
\hline \multirow[t]{4}{*}{ IR 01364-1042 } & 1.4 & $15.8 \pm 0.7$ & d \\
\hline & 4.8 & 11.8 & o \\
\hline & 8.4 & $8.2 \pm 0.4$ & $\mathrm{e}$ \\
\hline & 22.5 & $3.97 \pm 0.19$ & $\mathrm{~b}$ \\
\hline \multirow[t]{5}{*}{ IIIZw 35} & 1.4 & $40.6 \pm 1.3$ & d \\
\hline & 4.8 & 26.3 & $\mathrm{~g}$ \\
\hline & 8.4 & 19.7 & $\mathrm{e}$ \\
\hline & 15 & $10.0 \pm 1$ & $\mathrm{c}$ \\
\hline & 22.5 & $9.7 \pm 0.25$ & $\mathrm{~b}$ \\
\hline \multirow[t]{3}{*}{ UGC 2369} & 1.4 & $49 \pm 1.5$ & d \\
\hline & 8.4 & 13.3 & $\mathrm{e}$ \\
\hline & 22.5 & $5.3 \pm 0.3$ & a \\
\hline \multirow[t]{4}{*}{ IR $03359+1523^{+}$} & 1.4 & $19.4 \pm 0.7$ & d \\
\hline & 4.8 & 11.7 & g \\
\hline & 8.4 & 11.0 & $\mathrm{e}$ \\
\hline & 22.5 & $4.5 \pm 0.3$ & a \\
\hline \multirow[t]{4}{*}{ NGC 1614} & 1.4 & $137.1 \pm 4.9$ & d \\
\hline & 4.8 & $63 \pm 11$ & $\mathrm{j}$ \\
\hline & 8.4 & $40.7 \pm 0.3$ & $\mathrm{c}$ \\
\hline & 22.5 & $21.0 \pm 1$ & a \\
\hline \multirow[t]{4}{*}{ IR 05189-2524 } & 1.4 & $28.8 \pm 1.0$ & d \\
\hline & 4.8 & $17.0 \pm 0.9$ & $\mathrm{n}$ \\
\hline & 8.4 & 11.4 & $\mathrm{e}$ \\
\hline & 15 & $7.8 \pm 0.2$ & $\mathrm{c}$ \\
\hline \multirow[t]{5}{*}{ NGC 2623} & 1.4 & $95.7 \pm 2.9$ & d \\
\hline & 2.4 & $83.0 \pm 4$ & 1 \\
\hline & 4.8 & $59.0 \pm 10$ & $\mathrm{~h}$ \\
\hline & 8.4 & 35.5 & $\mathrm{e}$ \\
\hline & 22.5 & $18.3 \pm 0.2$ & $\mathrm{a}$ \\
\hline \multirow[t]{4}{*}{ IR $08572+3915$} & 1.4 & $4.3 \pm 0.4$ & d \\
\hline & 4.8 & $4.4 \pm 0.2$ & $\mathrm{n}$ \\
\hline & 8.4 & $4.1 \pm 0.2$ & $\mathrm{e}$ \\
\hline & 22.5 & $3.18 \pm 0.30$ & $\mathrm{~b}$ \\
\hline \multirow[t]{3}{*}{ UGC 4881} & 1.4 & $36.8 \pm 1.2$ & d \\
\hline & 8.4 & 8.8 & $\mathrm{e}$ \\
\hline & 22.5 & $3.5 \pm 0.5$ & $\mathrm{a}$ \\
\hline
\end{tabular}

+ Only one source of a close pair emits in the radio. ${ }^{*}$ Source possibly variable in radio. $1.4,4.8,8.4,15$ and $22.5 \mathrm{GHz}$ fluxes all made on the same date. References. (a) this work; (b) Prouton et al. (2004); (c) reduced from VLA archive. See Table 3 for details; (d) NVSS, Condon et al. (1998); (e) Condon et al. (1991a); (f) Sopp \& Alexander (1991); (g) Sopp \& Alexander (1992); (h) Gregory \& Condon (1991); (i) Griffith et al. (1994); (j) Griffith et al. (1995); (k) Condon et al. (1983); (l) Dressel \& Condon (1978); (m) Condon et al. (1991b); (n) Rush et al. (1996); (o) Baan \& Klöckner (2006); (p) Crawford et al. (1996); (q) Sramek \& Tovmassian (1976); (r) Imanishi et al. (2006); (s) Zhao et al. (1996); (t) Rodriguez-Rico et al. (2005); (u) Anantharamaiah et al. (2000); (v) Douglas et al. (1996); (w) Ficarra et al. (1985).
Table A.1. continued.

\begin{tabular}{|c|c|c|c|}
\hline Source & $\begin{array}{c}\text { Frequency } \\
\mathrm{GHz}\end{array}$ & $\begin{array}{l}\text { Flux } \\
\text { mJy }\end{array}$ & Ref. \\
\hline \multirow[t]{7}{*}{ UGC 5101} & 0.151 & $750 \pm 60$ & $\mathrm{f}$ \\
\hline & 1.4 & $170.1 \pm 5.8$ & $\mathrm{~d}$ \\
\hline & 4.8 & $73 \pm 15$ & f \\
\hline & 8.4 & 52.6 & $\mathrm{e}$ \\
\hline & 15 & $30 \pm 10$ & $\mathrm{f}$ \\
\hline & 22.5 & $18.0 \pm 1$ & $\mathrm{a}$ \\
\hline & 85.5 & 6 & $\mathrm{r}$ \\
\hline \multirow[t]{5}{*}{ IR $10173+0828$} & 1.4 & $9.9 \pm 0.9$ & $\mathrm{~d}$ \\
\hline & 4.8 & $5.88 \pm 0.2$ & $\mathrm{c}$ \\
\hline & 8.4 & 5.4 & $\mathrm{e}$ \\
\hline & 15 & $3.1 \pm 0.3$ & $\mathrm{c}$ \\
\hline & 22.5 & $2.9 \pm 0.4$ & $\mathrm{a}$ \\
\hline \multirow[t]{3}{*}{ IR $10565+2448$} & 1.4 & $57.0 \pm 2.1$ & $\mathrm{~d}$ \\
\hline & 4.8 & $22.21 \pm 0.13$ & $\mathrm{p}$ \\
\hline & 8.4 & 14.1 & $\mathrm{e}$ \\
\hline \multirow[t]{5}{*}{ Arp 148} & 1.4 & $36.4 \pm 1.2$ & $\mathrm{~d}$ \\
\hline & 4.8 & $14.0 \pm 0.5$ & $\mathrm{c}$ \\
\hline & 8.4 & $11.76 \pm 1$ & $\mathrm{a}$ \\
\hline & 15 & $8.0 \pm 1$ & $\mathrm{c}$ \\
\hline & 22.5 & $6.1 \pm 0.3$ & $\mathrm{a}$ \\
\hline \multirow{3}{*}{ UGC 6436} & 1.4 & $19.4 \pm 0.7$ & $\mathrm{~d}$ \\
\hline & 8.4 & $5.2 \pm 0.3$ & $\mathrm{a}$ \\
\hline & 22.5 & $2.8 \pm 0.5$ & $\mathrm{a}$ \\
\hline \multirow[t]{6}{*}{ Arp 299} & 0.365 & $1550 \pm 50$ & $\mathrm{v}$ \\
\hline & 1.4 & $686.3 \pm 25.4$ & d \\
\hline & 4.8 & $403 \pm 45$ & $\mathrm{~h}$ \\
\hline & 8.4 & $243 \pm 8$ & $\mathrm{c}$ \\
\hline & 15 & $160 \pm 3$ & $\mathrm{c}$ \\
\hline & 22.5 & $74 \pm 2$ & $\mathrm{c}$ \\
\hline \multirow[t]{5}{*}{ IR $12112+0305$} & 1.4 & $23.3 \pm 0.8$ & $\mathrm{~d}$ \\
\hline & 4.8 & $14.7 \pm 0.2$ & $\mathrm{c}$ \\
\hline & 8.4 & 10.0 & $\mathrm{e}$ \\
\hline & 15 & $5.7 \pm 0.2$ & $\mathrm{c}$ \\
\hline & 22.5 & $5.7 \pm 0.3$ & $\mathrm{a}$ \\
\hline \multirow[t]{6}{*}{ UGC $08058^{*}$} & 0.365 & $551 \pm 39$ & $\mathrm{v}$ \\
\hline & 1.4 & $274 \pm 3$ & $\mathrm{c}$ \\
\hline & 4.8 & $265 \pm 1$ & $\mathrm{c}$ \\
\hline & 8.4 & $189 \pm 1$ & $\mathrm{c}$ \\
\hline & 15 & $146 \pm 1$ & $\mathrm{c}$ \\
\hline & 22.5 & $137 \pm 2$ & $\mathrm{c}$ \\
\hline \multirow[t]{5}{*}{ UGC 8387} & 1.4 & $104.4 \pm 3.2$ & $\mathrm{~d}$ \\
\hline & 4.8 & 46 & $\mathrm{~m}$ \\
\hline & 8.4 & 34.9 & $\mathrm{e}$ \\
\hline & 15 & $12 \pm 2$ & $\mathrm{c}$ \\
\hline & 22.5 & $7.5 \pm 2$ & $\mathrm{a}$ \\
\hline \multirow[t]{6}{*}{ NGC 5256} & 0.365 & $353 \pm 43$ & $\mathrm{v}$ \\
\hline & 1.4 & $126.3 \pm 4.5$ & d \\
\hline & 4.8 & $43.3 \pm 3.1$ & $\mathrm{n}$ \\
\hline & 8.4 & $24.1 \pm 1$ & $\mathrm{c}$ \\
\hline & 15 & $21.0 \pm 1$ & $\mathrm{c}$ \\
\hline & 20.0 & $14.9 \pm 1.5$ & $\mathrm{n}$ \\
\hline \multirow[t]{9}{*}{ UGC 8696} & 0.151 & $590 \pm 60$ & $\mathrm{f}$ \\
\hline & 0.365 & $335 \pm 37$ & $\mathrm{v}$ \\
\hline & 1.4 & $144.7 \pm 5.1$ & d \\
\hline & 2.7 & $96 \pm 6$ & $q$ \\
\hline & 4.8 & $71 \pm 15$ & $\mathrm{f}$ \\
\hline & 8.4 & 43.5 & e \\
\hline & 15 & $29.5 \pm 1$ & $\mathrm{c}$ \\
\hline & 22.5 & $14.3 \pm 0.3$ & $\mathrm{a}$ \\
\hline & 85.6 & 6 & $\mathrm{r}$ \\
\hline \multirow[t]{3}{*}{ IR $14348-1447$} & 1.4 & $35.9 \pm 1.2$ & d \\
\hline & 8.4 & $10.8 \pm 1$ & $\mathrm{a}$ \\
\hline & 22.5 & $4.5 \pm 0.5$ & $\mathrm{a}$ \\
\hline
\end{tabular}


M. S. Clemens et al.: Modeling the spectral energy distribution of ULIRGs. I., Online Material $p 7$

Table A.1. continued.

\begin{tabular}{|c|c|c|c|}
\hline Source & $\begin{array}{c}\text { Frequency } \\
\text { GHz }\end{array}$ & $\begin{array}{l}\text { Flux } \\
\text { mJy }\end{array}$ & $\overline{\text { Ref. }}$ \\
\hline \multirow[t]{5}{*}{ IZw 107} & 0.408 & $100 \pm 20$ & $\mathrm{~W}$ \\
\hline & 1.4 & $50.3 \pm 1.6$ & d \\
\hline & 4.8 & $32.0 \pm 0.6$ & $\mathrm{~h}$ \\
\hline & 8.4 & $14.3 \pm 1$ & $\mathrm{a}$ \\
\hline & 22.5 & $3.6 \pm 0.3$ & $\mathrm{a}$ \\
\hline \multirow[t]{6}{*}{ IR $15250+3609$} & 0.151 & $<100$ & $\mathrm{f}$ \\
\hline & 1.4 & $14.5 \pm 0.6$ & d \\
\hline & 4.8 & $13 \pm 3$ & $\mathrm{f}$ \\
\hline & 8.4 & 10.5 & $\mathrm{e}$ \\
\hline & 15 & $8 \pm 3$ & $\mathrm{f}$ \\
\hline & 22.5 & $4.2 \pm 0.3$ & $\mathrm{a}$ \\
\hline \multirow[t]{11}{*}{ Arp 220} & 0.151 & $260 \pm 30$ & $\mathrm{f}$ \\
\hline & 0.365 & $435 \pm 26$ & $\mathrm{v}$ \\
\hline & 1.4 & $326.3 \pm 9.8$ & d \\
\hline & 2.4 & $312 \pm 16$ & 1 \\
\hline & 2.7 & $260 \pm 13$ & $\mathrm{k}$ \\
\hline & 4.8 & $210 \pm 20$ & $\mathrm{f}$ \\
\hline & 8.4 & 148.0 & $\mathrm{e}$ \\
\hline & 15 & $110 \pm 1$ & $\mathrm{f}$ \\
\hline & 22.5 & $90 \pm 6$ & $\mathrm{~s}$ \\
\hline & 43 & $44 \pm 4$ & $\mathrm{t}$ \\
\hline & 97.2 & $61 \pm 10$ & $\mathrm{u}$ \\
\hline \multirow[t]{5}{*}{ NGC 6286} & 0.365 & $431 \pm 37$ & $\mathrm{v}$ \\
\hline & 1.4 & $156.7 \pm 5.6$ & d \\
\hline & 4.8 & $53.0 \pm 8$ & $\mathrm{~h}$ \\
\hline & 8.4 & $31.5 \pm 1.6$ & $\mathrm{a}$ \\
\hline & 22.5 & $11.2 \pm 1.5$ & $\mathrm{a}$ \\
\hline \multirow[t]{5}{*}{ NGC 7469} & 0.365 & $397 \pm 24$ & $\mathrm{v}$ \\
\hline & 1.4 & $180.5 \pm 5.4$ & d \\
\hline & 4.8 & $70 \pm 8$ & $\mathrm{~g}$ \\
\hline & 8.4 & $50.2 \pm 0.6$ & $\mathrm{c}$ \\
\hline & 22.5 & $17.50 \pm 0.5$ & b \\
\hline \multirow[t]{4}{*}{ IC 5298} & 1.4 & $34.7 \pm 1.4$ & d \\
\hline & 4.8 & 13.9 & $\mathrm{~g}$ \\
\hline & 8.4 & $8.2 \pm 0.4$ & $\mathrm{e}$ \\
\hline & 22.5 & $3.86 \pm 0.18$ & $\mathrm{~b}$ \\
\hline \multirow[t]{4}{*}{ Mrk 331} & 1.4 & $70.7 \pm 2.2$ & d \\
\hline & 4.8 & 31.1 & $\mathrm{~g}$ \\
\hline & 8.4 & $22.3 \pm 1$ & $\mathrm{a}$ \\
\hline & 22.5 & $10.0 \pm 0.3$ & $\mathrm{~b}$ \\
\hline
\end{tabular}

